Pure and Applied Mathematics Quarterly

Volume 5, Number 2

(Special Issue: In honor of

Friedrich Hirzebruch, Part 1 of 2)

$781-852,2009$

\title{
Katz's Middle Convolution Algorithm
}

\author{
Carlos Simpson
}

\begin{abstract}
This is an expository account of Katz's middle convolution operation on local systems over $\mathbf{P}^{1}-\left\{q_{1}, \ldots, q_{n}\right\}$. We describe the Betti and de Rham versions, and point out that they give isomorphisms between different moduli spaces of local systems, following Völklein, Dettweiler-Reiter, Haraoka-Yokoyama. Kostov's program for applying the Katz algorithm is to say that in the range where middle convolution no longer reduces the rank, one should give a direct construction of local systems. This has been done by Kostov and Crawley-Boevey. We describe here an alternative construction using the notion of cyclotomic harmonic bundles: these are like variations of Hodge structure except that the Hodge decomposition can go around in a circle.
\end{abstract}

Keywords: Connection, Fundamental group, Representation, Middle convolution, Logarithmic de Rham cohomology, Higgs bundle

\section{INTRODUCTION}

There is a growing body of literature about Katz's "middle convolution" algorithm on local systems on $\mathbb{P}^{1}-\left\{q_{1}, \ldots, q_{n}\right\}$. The purpose of the present paper is expository: we would like to describe two versions of Katz's construction in complex geometry, the Betti version involving complex local systems, and the de Rham version involving vector bundles with logarithmic connection. Katz's book [86] was written in the framework of $\ell$-adic sheaves, which at first made it difficult to understand for complex geometers including myself. Subsequently, Völklein

Received September 22, 2006. 
and Dettweiler-Reiter recast the construction in complex geometry and algebra. In $\S \S 2.7-2.9$ of Katz's book, the convolution was defined in a geometric way which is applicable in any context where one has a Grothendieck formalism and a category of perverse sheaves. Thus, the translation into complex geometry may be viewed as coming directly from there [86, 5.9]. Katz then interpreted the convolution as conjugate to a tensor product, via Fourier transform, and used that to obtain some of the main properties of his construction. The complex analogy for this would a priori bring into play the notion of irregular connections on a 2-dimensional variety, a theory which remains poorly understood (see however [15] and the recent preprint [3]). It is possible to do a full treatment of middle convolution staying within the realm of complex geometry but without using Fourier transform, as has been shown and exploited by the works of Strambach, Völklein, Dettweiler, Reiter, Kostov, Crawley-Boevey, Haraoka, Yokoyama.

Many applications of Katz's theory concern the case of rigid local systems. For example, Gleizer has studied explicit solutions [55], and Roberts' preprint [123] includes an extensive discussion of how to apply the algorithm to determine which rigid local systems exist. Völklein, Dettweiler and Reiter have done extensive work on using Katz's existence results in the rigid case to construct motivic local systems with interesting monodromy groups, obtaining results on the inverse Galois problem.

The middle convolution transformation was first applied in the non-rigid case by Kostov. An important invariant which we denote by $\delta(\vec{g})$ is the change in rank induced by Katz's transformation. As long as $\delta(\vec{g})<0$ we can apply middle convolution to reduce the rank (or otherwise, conclude that the local system couldn't exist). Kostov made the fundamental observation that when we get into the range $\delta(\vec{g}) \geq 0$, we should expect that the local system always exists and look for a direct construction. Kostov applied this to solve the existence problem in many cases [89]-[95], such as when $\vec{g}$ is simple i.e. the multiplicities of eigenvalues are not all divisible by the same integer $d>1$, or for generic eigenvalues even if $\vec{g}$ is not simple [92]. Crawley-Boevey looked at the existence question from a point of view of root systems in [34] where Katz's algorithm plays a role. In that language, the transformation on local monodromy data is considered as a reflection in a root system, and a sequence of reflections is used to move up to the positive chamber. Once we are in the positive chamber, analogous to the condition $\delta(\vec{g}) \geq 0$, Crawley-Boevey found a direct construction 
of indecomposable parabolic bundles, and applied a parabolic variant of Weil's theorem to construct flat connections.

At the end of the present paper, we propose a technique for constructing local systems in the range $\delta(\vec{g}) \geq 0$ by using the correspondence between Higgs bundles and local systems in the parabolic case. This construction is heavily inspired by Kostov's program, and is obviously a variant on Crawley-Boevey's indecomposable parabolic bundles. So, it is not really very new but might present some advantages such as making clear the role played by the condition $\delta(\vec{g}) \geq 0$. The objects we introduce, cyclotomic harmonic bundles, might be interesting in their own right such as for studying the behavior of everything near infinity in the moduli spaces.

We will look at Katz's operation as giving an isomorphism between moduli spaces for different local monodromy data. Of course this applies to the rigid case too, but as Katz pointed out long ago, for local systems on $\mathbb{P}^{1}$ with specified singularities, local rigidity implies the stronger rigidity statement that there is at most one irreducible representation with the given local data. Thus, in the rigid case the moduli spaces are single points so even the cardinality is not an interesting invariant. Instead, we are motivated by looking for low-dimensional moduli spaces, for which things like Hitchin's hyperkähler structure, or the RiemannHilbert correspondence, could be viewed explicitly. The phrase "toy example" was coined by T. Hausel in [65] to refer to this kind of low-dimensional case arising from a punctured projective line. He looked at a space of parabolic Higgs bundles of rank 2 on $\mathbb{P}^{1}-\left\{q_{1}, \ldots, q_{4}\right\}$. Boalch looked at an example of the middle convolution relating this space to a space of rank 3 representations in [14] [15], and considered the Painlevé equations for these cases. It seems like a good idea to pursue the philosophy of looking at low-dimensional cases, and to get started we need to have a thorough understanding of how the classification based on Katz's algorithm works. That's the motivation for this paper.

Conceptually, the middle convolution operation is pretty easy to understand. Let $Y$ and $Z$ denote two copies of the projective line $\mathbb{P}^{1}$, with reduced effective divisors $Q_{Y} \subset Y$ and $Q_{Z} \subset Z$ (which we will often denote just by $Q$ ), both given by the same finite collection of $n$ points $Q=\left\{q_{1}, \ldots, q_{n}\right\}$. Let $D \subset Z \times Y$ denote the "diagonal configuration" consisting of the diagonal $\Delta$ plus the vertical and horizontal divisors given by preprojections of $Q$. 
A convoluter is a rank one local system on $Z \times Y$ with singularities along $D$. In Katz's original setup this would be a rank one $\ell$-adic sheaf. In the complex geometric "Betti" and "de Rham" situations we consider here, the convolution object is respectively a rank one representation of $\pi_{1}(Z \times Y-D)$, or a logarithmic connection on the trivial bundle given by a logarithmic one-form. In either case we denote the convoluter by $\beta$. Let

$$
\xi:((Z \times Y)-D) \longrightarrow\left(Z-Q_{Z}\right), \quad \eta:((Z \times Y)-D) \longrightarrow\left(Y-Q_{Y}\right)
$$

denote the two projections. Given an irreducible rank $r$ local system $L$ on $Y-Q$, we can form the "raw convolution" defined as the higher direct image

$$
R C_{\beta}(L):=\mathbb{R}^{1} \xi_{*}\left(\eta^{*}(L) \otimes \beta\right),
$$

a local system on $Z-Q_{Z}$. Unfortunately, the raw convolution will not in general be an irreducible local system, because there are some contributions whenever the tensor product $\eta^{*}(L) \otimes \beta$ has trivial eigenvalues along the "horizontal" piece $H:=$ $\eta^{*}\left(Q_{Y}\right)$ of the divisor $D$. This is remedied by defining the "middle convolution" to be the middle direct image

$$
M C_{\beta}(L):=M \mathbb{R}^{1} \xi_{*}\left(\eta^{*}(L) \otimes \beta\right),
$$

heuristically defined as the kernel of the map to the quotient systems corresponding to the unwanted local cohomolgy groups.

When studying local systems on $\mathbb{P}^{1}$ with singularities, we are interested in fixing the local type of the singularities. For the present paper, we will simplify things considerably by making the convention that the local monodromy transformations be semisimple (Convention 2.1) or the corresponding statement for the residues of a logarithmic connection (Convention 2.2). This allows us to avoid complicated discussions of Jordan normal forms. The reader who is interested may refer to the original references for discussions of this aspect.

When we discuss moduli spaces we will consider the moduli spaces of local systems with fixed conjugacy classes of local monodromy. The notion of rigidity considered by Katz takes into account the fixing of the local conjugacy classes. So, the first and in some sense main question about Katz's construction is to understand what is its effect on the local monodromy transformations.

We will try to explain the answer, and how to see why it works that way. This will occupy most of the paper, and is the main subject of our exposition. Of 
course it has already been treated by Katz in the $\ell$-adic case, and by Strambach, Völklein, Dettweiler, Reiter, Kostov, Crawley-Boevey, Haraoka, Yokoyama and others in the complex case. Thus there is nothing new in our exposition. We hope it will be useful as an explanation allowing readers more easily to consult the original references.

At the end of the paper, we consider the question of how to construct local systems in the range $\delta(\vec{g}) \geq 0$. We propose a construction which is based on Donaldson-style Yang-Mills theory [49] [70], in which we construct a polystable Higgs bundle with parabolic structure corresponding to the local monodromy. This is similar to the construction of systems of Hodge bundles which was used in [130]. Here we introduce a new notion which makes the problem much easier: cyclotomic harmonic bundles. These are harmonic bundles which are fixed under the action of a finite cyclic subgroup $\mu_{m} \subset \mathbb{C}^{*}$, for the usual action of $\mathbb{C}^{*}$ on the space of Higgs bundles. These are related to the Higgs bundles considered by Hitchin in [72]. A cyclotomic harmonic bundle is very much like a variation of Hodge structure, in that the bundle decomposes $E=\bigoplus_{p=0}^{m-1} E^{p}$. The only difference is that the indexation is really by $p \in \mu_{m}^{*} \cong \mathbb{Z} / m \mathbb{Z}$, and the KodairaSpencer components of the Higgs field go between $E^{p}$ and $E^{p-1} \otimes \Omega_{X}^{1}$ where $p-1$ is taken modulo $m$. Thus $\theta$ is no longer necessarily nilpotent. Our construction takes place in the maximal case when $m=r$ is the rank of $E$, and $\theta$ is not nilpotent. This means that the $E^{p}$ are line bundles. Thus the description of $(E, \theta)$ is elementary. It turns out that incorporating parabolic structures into the picture in order to obtain a required local monodromy type, the condition $\delta(\vec{g}) \geq 0$ is exactly what is needed for the degrees of the line bundles to work out correctly and enable the construction. Unfortunately it doesn't work when the dimension of the moduli space is 2 .

In the last section we discuss some questions and directions for further study.

Acknowledgements: I would specially like to thank V. Kostov for many helpful discussions, and for a particularly illuminating talk many years ago in which he explained his utilisation of Katz's algorithm. Also I would like to thank O. Gleizer for some interesting discussions a while ago. At Princeton in 2005, Deligne and Katz raised the question of how to understand what is going on, which prompted the present write-up. Many aspects we consider here, such as moduli spaces with 
fixed conjugacy classes, and logarithmic connections, showed up in the course of recent joint works with K. Corlette and J. Iyer.

\section{Connections And LOCAL Systems}

Denote by $Y$ a smooth projective curve, with $K \subset Y$ a reduced divisor. Write $K=k_{1}+\ldots+k_{n}$, with points $k_{i} \in Y$. We consider local systems $L$ over $Y-K$. If $x \in Y-K$ is a choice of basepoint then a local system corresponds to the monodromy representation

$$
\rho: \pi_{1}(Y-K, x) \rightarrow G L\left(L_{x}\right) .
$$

The local monodromy transformations are the $\rho\left(\alpha_{i}\right)$ where $\alpha_{i}$ is a loop in standard form going from $x$ to near $k_{i}$, once around clockwise, then back to $x$. In this paper, we will systematically make the convention :

Convention 2.1. The local monodromy transformations are semisimple, i.e. diagonalizable matrices.

A logarithmic connection on $(Y, K)$ is a vector bundle $E$ on $Y$, with a connection operator

$$
\nabla: E \rightarrow E \otimes \Omega_{Y}^{1}(\log K) .
$$

The monodromy of $(E, \nabla)$ is a local system on $Y-K$, described for example as the sheaf $L=E^{\nabla}$ of analytic holomorphic sections $e$ of $E$ with $\nabla(e)=0$. Over $Y-K$ this is a locally constant sheaf or a local system, and corresponds to a monodromy representation $\rho_{E, \nabla}$.

The residue of $(E, \nabla)$ at a point $k_{i} \in K$ is the pair $\left(E_{k_{i}}, \operatorname{res}\left(\nabla, k_{i}\right)\right)$ consisting of the fiber of $E$ over $k_{i}$, and the residue of the connection which is an endomorphism of the fiber.

We say that "the residues of $\nabla$ are semisimple" if these endomorphisms are semisimple i.e. diagonalizable. Furthermore, in order to insure that the monodromy transformation satisfies Convention 2.1, it is convenient to ask that the eigenvalues of the residues never differ by integers. Indeed, if there are pairs of eigenvalues differing by integers even in a semisimple residue, this can typically lead to Jordan blocks of size $>1$ in the monodromy. This gives the analogous convention for the de Rham case. 
Convention 2.2. The residues of $\nabla$ are semisimple and their eigenvalues don't differ by nonzero integers.

These conventions greatly reduce the complexity of the notation and arguments required to understand Katz's constructions. Of course Katz and subsequent authors all considered the more general case of arbitrary Jordan normal forms, and we refer the reader to those references for a more in-depth look at this aspect.

2.1. Middle cohomology. If the residues don't have integer eigenvalues, then Deligne's theory gives an easy description of the cohomology of the monodromy local system. We denote by $D R(Y, E)$ the logarithmic de Rham complex

$$
D R(Y, E):=\left[E \stackrel{\nabla}{\rightarrow} E \otimes \Omega_{Y}^{1}(\log K)\right] .
$$

The connection $\nabla$ and the divisor $K$ are missing and implicit in this notation. This abuse allows us to shorten most displays below. Unless otherwise stated, all de Rham complexes are supposed to be logarithmic with respect to the relevant divisor.

Proposition 2.3. Suppose that the eigenvalues of the residues of $(E, \nabla)$ are never integers. Let $L_{\rho}:=E^{\nabla}$ be the monodromy local system on $Y-K$. Then hypercohomology of the logarithmic de Rham complex $D R(Y, E)$ calculates the cohomology of $Y-K$ with coefficients in $L_{\rho}$ :

$$
\mathbb{H}^{\cdot} D R(Y, E) \sim H^{\cdot}\left(Y-K, L_{\rho}\right) .
$$

In the case where some eigenvalues are integers, the situation is more complicated. The same monodromy representation can come from several different logarithmic connections, whose residual eigenvalues will differ by integers. The cohomology which is calculated by the de Rham complex will in principle depend on which lift we have chosen. A canonical choice, somewhat different from the choices coming from lifts, is given by the notion of "middle cohomology" and the "middle de Rham complex". The reason for the word "middle" is that it corresponds to the middle perversity in intersection cohomology. In the onedimensional case, as was well understood by Katz, the notion of intersection cohomology corresponds to the more classical construction $j_{*}$, as opposed to the derived $\mathbb{R} j_{*}$. In this context the word "middle" is more notation than notion. 
The Betti version is as follows. Let $j: Y-K \hookrightarrow Y$ denote the inclusion. Assume $K$ is nonempty. If $\rho$ is a representation of the fundamental group of $Y-K$ corresponding to a local system $L_{\rho}$ on $Y-J$, then we define the middle cohomology

$$
M H^{i}\left(Y, L_{\rho}\right):=H^{i}\left(Y, j_{*}\left(L_{\rho}\right)\right) .
$$

The non-derived $j_{*}\left(L_{\rho}\right)$ is the degree zero part of the total derived $\mathbb{R} j_{*}\left(L_{\rho}\right)$; the other piece is $R^{1} j_{*}\left(L_{\rho}\right)[-1]$. Which gives an exact triangle in the derived category

$$
j_{*}\left(L_{\rho}\right) \rightarrow \mathbb{R} j_{*}\left(L_{\rho}\right) \rightarrow R^{1} j_{*}\left(L_{\rho}\right)[-1] \rightarrow j_{*}\left(L_{\rho}\right)[1] \ldots
$$

Look at the long exact sequence of hypercohomology for this triangle. The hypercohomology of the total $\mathbb{R} j_{*}$ gives the cohomology of $Y-K$. Also $R^{1} j_{*}\left(L_{\rho}\right)$ is concentrated at $K$, so it only contributes for global sections. In particular we have

$$
M H^{0}\left(Y, L_{\rho}\right)=H^{0}\left(Y-K, L_{\rho}\right)
$$

and there is a long exact sequence

$$
0 \rightarrow M H^{1}\left(Y, L_{\rho}\right) \rightarrow H^{1}\left(Y-K, L_{\rho}\right) \rightarrow \oplus_{k \in K} R^{1} j_{*}\left(L_{\rho}\right)_{k} \rightarrow M H^{2}\left(Y, L_{\rho}\right) \rightarrow 0 .
$$

Since $K$ is nonempty, $Y-K$ is homotopic to a one dimensional complex so its $H^{2}$ with local coefficients vanishes.

An observation which is important for defining the middle convolution is the following.

Proposition 2.4. Suppose that for at least one point $k_{i} \in K$, the local monodromy has no fixed vectors. Then the middle cohomology in degrees zero and two vanishes.

Proof: It is clear that $H^{0}\left(Y, L_{\rho}\right)=0$ because there are no flat sections near the point $k_{i}$, so there can be no global flat sections. This proves that $M H^{0}=0$. There is a Poincaré-Verdier duality between $M H^{i}(Y, L)$ and $M H^{2-i}\left(Y, L^{*}\right)$, and $L^{*}$ also has no fixed vectors at $k_{i}$. This gives $M H^{2}(Y, L)=0$. One can also prove the vanishing by a direct topological argument.

As a corollary we obtain the dimension of the middle cohomology group in this case: 
Corollary 2.5. Suppose $L_{\rho}$ is a local system of rank $r$ on $Y-K$, and suppose that for at least one point $k_{i} \in K$ the local monodromy has no fixed vectors. Then we have

$$
\operatorname{dim} M H^{1}\left(Y, L_{\rho}\right)=r(n-2)-\sum_{j=1}^{n} \operatorname{cofix}\left(L_{\rho}, k_{j}\right) .
$$

where $n$ is the number of points in $K$ and $\operatorname{cofix}\left(L_{\rho}, k_{j}\right)$ is the dimension of the space of cofixed vectors of the local monodromy at $k_{i}$.

Proof: Note that $H^{0}\left(Y-K, L_{\rho}\right)=0$ as pointed out in the proof of 2.4, and $H^{2}\left(Y-K, L_{\rho}\right)=0$ because $Y-K$ is homotopically a 1 -dimensional complex. Thus by calculating the Euler characteristic we have

$$
\operatorname{dim} H^{1}\left(Y-K, L_{\rho}\right)=r(n-2) .
$$

On the other hand,

$$
\operatorname{dim} H^{1}\left(B_{k_{j}}^{*}, L_{\rho}\right)=\operatorname{cofix}\left(L_{\rho}, k_{j}\right)
$$

The exact sequence 2.1 gives the dimension of $M H^{1}$.

2.2. Middle homology. The middle homology is obtained by duality with the middle cohomology:

$$
M H_{i}(Y-K, L):=M H^{i}\left(Y-K, L^{*}\right)^{*} .
$$

This is interesting only if the monodromy of $L$ has some eigenvalues equal to 1 around a point $k_{i}$. The loop around that point, with the eigenvector as coefficient, gives a cycle in $H_{1}(Y-K, L)$.

Let $F_{i} \subset L_{z}$ denote the subspace of vectors fixed by the monodromy transformation $\rho_{L}\left(\alpha_{i}\right)$. Since we are assuming that the local monodromy transformations are unipotent, the dimension of $F_{i}$ is equal to the multiplicity of 1 as eigenvalue of $\rho_{L}\left(\alpha_{i}\right)$. We get a map

$$
\phi: \bigoplus_{i=1}^{k} F_{i} \rightarrow H_{1}(Y-K, L) .
$$

Lemma 2.6. Suppose that the monodromy around at least one of the points $k_{i}$ has no fixed vector. Then $\phi$ is injective and the first middle homology is the cokernel of the map $\phi$ :

$$
M H_{1}(\Gamma, L):=\frac{H_{1}(Y-K, L)}{\phi \bigoplus_{i=1}^{k} F_{i}} .
$$


Proof: This is dual to Proposition 2.4.

2.3. Middle de Rham cohomology. For the de Rham version of middle cohomology, if $(E, \nabla)$ is a vector bundle with logarithmic connection on $(Y, K)$, we define the middle de Rham complex

$$
M D R(Y, E, \nabla)=\left[M D R^{0}(Y, E, \nabla) \rightarrow M D R^{1}(Y, E, \nabla)\right]
$$

with

$$
\operatorname{MDR}^{0}(Y, E, \nabla):=E
$$

and

$$
M D R^{1}(E, \nabla):=\operatorname{ker}\left(E \otimes \Omega_{Y}^{1}(\log K) \rightarrow E_{K}^{0}\right)
$$

where $E_{K}^{0}$ is the quotient of the fiber $E_{K}$ over $K$, corresponding to the 0eigenspaces of $\operatorname{res}\left(\nabla, k_{i}\right)$ at the points $k_{i} \in K$. The differential is given by $\nabla$ as for the usual de Rham complex.

We should stress here that this definition is the right one only under our convention and assumption that the local monodromy, and the residues of $\nabla$, are semisimple.

Define the middle de Rham cohomology to be the hypercohomology $\mathbb{H}^{i} M D R(Y, E, \nabla)$.

By definition we have a short exact sequence of complexes of sheaves on $Y$,

$$
0 \rightarrow M D R(Y, E, \nabla) \rightarrow D R(Y, E, \nabla) \rightarrow E_{K}^{0}[-1] \rightarrow 0 .
$$

This gives the same kind of long exact sequence as before.

Lemma 2.7. Suppose that the residues of $\nabla$ have no nonzero integer eigenvalues. Then the above short exact sequence for the middle de Rham cohomology coincides after Riemann-Hilbert correspondence, with the previous exact triangle for the middle Betti cohomology. In particular, if $L_{\rho}$ is the monodromy local system $E^{\nabla}$ then we have a natural isomorphism

$$
\mathbb{H}^{i} M D R(Y, E, \nabla) \cong M H^{i}\left(Y, L_{\rho}\right) .
$$

If there are nonzero integer eigenvalues, on the other hand, then the corresponding subspaces are fixed for the monodromy transformation but don't appear in the quotient $E_{K}^{0}$. In this case the middle de Rham cohomology will be different from the middle Betti cohomology. 
Exercise 1. Describe what cohomology is calculated by $D R(Y, E, \nabla)$ and $M D R$ $(Y, E, \nabla)$ when the residues of $\nabla$ may have some nonzero integer eigenvalues.

Hint: It depends on the sign of the eigenvalues.

Proposition 2.4 above thus has the corresponding corollary in the de Rham case.

Corollary 2.8. Suppose that the residues of $\nabla$ are semisimple and have no nonzero integer eigenvalues, and suppose that for at least one point $k_{i} \in K$, the residue $\operatorname{res}\left(\nabla, k_{i}\right)$ has all eigenvalues different from 0 . Then the middle cohomology in degrees zero and two vanishes:

$$
\mathbb{H}^{0} M D R(Y, E, \nabla)=0, \quad \mathbb{H}^{2} M D R(Y, E, \nabla)=0 .
$$

The dimension of the middle cohomology in degree 1 is given by

$$
\operatorname{dim} M H^{1}\left(Y, L_{\rho}\right)=r(n-2)-\sum_{j=1}^{n} \operatorname{rk}\left(E_{k_{j}}^{0}\right) .
$$

Proof: This is immediate from 2.4 and 2.7, and with Corollary 2.5 or its proof we get the dimension count.

2.4. The Betti moduli spaces. We are interested in the moduli of representations with fixed conjugacy classes at the singularities. The first version to look at is the "Betti" moduli space. See [32].

Let $q_{1}, \ldots, q_{n}$ be $n$ distinct points in $Y:=\mathbb{P}^{1}$ and fix a basepoint $z$ different from these. Put $\Gamma:=\pi_{1}\left(Y-\left\{q_{1}, \ldots, q_{n}\right\}, z\right)$. Let $\gamma_{1}, \ldots, \gamma_{n}$ denote standard loops based at $z$ going around the points $q_{1}, \ldots, q_{n}$ respectively.

Fix closed subsets $C_{1}, \ldots, C_{n} \subset G L(r)=G L(r, \mathbb{C})$ invariant under the conjugation action. In the present paper in keeping with Convention 2.1 these will be semisimple conjugacy classes (see below). However the definition can be made with more general closed subsets which would then have to contain many different conjugacy classes including semisimple ones. In this case the structure of the moduli space is more complicated, for example it can be nonempty even when the moduli space for the semisimple conjugacy classes in the closure might be empty. 
Let

$$
\operatorname{Rep}\left(\Gamma, G L(r) ; C_{1} \ldots C_{n}\right) \subset \operatorname{Rep}(\Gamma, G L(r))
$$

be the closed subset of representations $\rho: \Gamma \rightarrow G L(r)$ such that

$$
\rho\left(\gamma_{i}\right) \in C_{i} .
$$

Since it is a closed subset of an affine variety, it is also affine. The group $G L(r)$ acts on the representation variety and it preserves our closed subset because we have assumed that the $C_{i}$ are conjugation-invariant. Thus we get an action of $G L(r)$ on the affine variety $\operatorname{Rep}\left(\Gamma, G L(r) ; C_{1} \ldots C_{n}\right)$ so we can take the universal categorical quotient

$$
M_{B}\left(C_{1}, \ldots, C_{n}\right):=\operatorname{Rep}\left(\Gamma, G L(r) ; C_{1} \ldots C_{n}\right) / G L(r) .
$$

This has the following usual description on the level of points. Two points $\rho, \rho^{\prime}$ of the representation variety are $S$-equivalent if the closures of their orbits intersect. In the preimage of any point of $M_{B}$ there is a unique closed orbit, which shows that this relation is an equivalence relation and the points of $M_{B}\left(C_{1}, \ldots, C_{n}\right)$ are the $S$-equivalence classes. We have the same description for the action of $G l(r)$ on $\operatorname{Rep}(\Gamma, G L(r))$, and since $\operatorname{Rep}\left(\Gamma, G L(r) ; C_{1} \ldots C_{n}\right)$ is a closed $G L(r)$-invariant subvariety, the closure of an orbit of $\rho \in \operatorname{Rep}(\Gamma, G L(r)$; $\left.C_{1} \ldots C_{n}\right)$ is the same when taken in the bigger representation variety or the closed subset. Therefore, the relation of $S$-equivalence when we restrict the conjugacy classes, is the restriction of this relation on the full variety. ${ }^{*}$ The relation of $S$-equivalence for the full representation variety is well-understood, see LubotskyMagid [103] for example. In particular, two points $\rho, \rho^{\prime}$ are $S$-equivalent if and only if their semisimplifications are isomorphic. The semisimplification is again a representation in $\operatorname{Rep}\left(\Gamma, G L(r) ; C_{1} \ldots C_{n}\right)$, and the points of $M_{B}\left(C_{1}, \ldots, C_{n}\right)$ represent the isomorphism classes of semisimple representations.

Now restrict our attention to the case of semisimple conjugacy classes $C_{1}, \ldots, C_{n}$, that is to say the conjugacy classes of diagonalizable matrices. The $C_{i} \subset G L(r)$ are closed subsets, so the above discussion applies.

It is convenient to think of a semisimple class as being determined by a divisor on $\mathbb{G}_{m}$. Write a divisor as $g=\sum_{a \in \mathbb{G}_{m}} m(a)[a]$ where $[a]$ is the point $a$ considered

${ }^{*}$ Notice that for this statement, we have used the condition that the $C_{i}$ are closed subsets. If we tried to do this with locally closed subsets, for example corresponding to nonsemisimple conjugacy classes but not their closures, it wouldn't work the same way. 
as a reduced effective divisor and the sum is finite. If $r=\operatorname{deg}(g):=\sum_{a} m(a)$, then the divisor $g$ corresponds to the conjugacy class $C(g) \subset G L(r)$ of diagonalizable matrices having eigenvalues $a$ with multiplicities $m(a)$. A sequence of semisimple conjugacy classes is then represented by a local monodromy vector of $n$ divisors $\vec{g}=\left(g_{1}, \ldots, g_{n}\right) \in \operatorname{Div}\left(\mathbb{G}_{m}\right)^{n}$. We come to our main notation for the Betti moduli spaces:

$$
M_{B}(\vec{g}):=M_{B}\left(C\left(g_{1}\right), \ldots, C\left(g_{n}\right)\right),
$$

where the collection of points $Q=q_{1}+\ldots+q_{n}$ is implicit but not mentionned.

The vector or partition consisting of the $m(a)$ is a partition of $r$. Kostov calls this the multiplicity vector, and the vector of multiplicity vectors corresponding to $g_{1}, \ldots, g_{n}$ is called by Kostov the polymultiplicity vector or PMV. To obtain a geographic understanding one should look only at the PMV, see Roberts [123].

2.5. Nitsure's de Rham moduli space. We can define the following 2-functor $\mathcal{M}_{D R}(r, d)$ of $\mathbb{C}$-schemes of finite type $T$. Put $\mathcal{M}_{D R}(r)[T]$ equal to the groupoid of $(E, \nabla)$ where $E$ is a vector bundle of rank $r$ and degree $d$ on $P \times T$ and

$$
\nabla: E \rightarrow E \otimes_{\mathcal{O}_{P \times T}} \Omega_{P \times T / T}^{1}(\log Q \times T)
$$

is a relative logarithmic connection. Standard moduli theory shows that it is an Artin algebraic stack locally of finite type.

Say that a logarithmic connection $(E, \nabla)$ is semistable if for any subbundle $F \subset E$ preserved by $\nabla$, we have

$$
\frac{\operatorname{deg}(F)}{\operatorname{rk}(F)} \leq \frac{\operatorname{deg}(E)}{\operatorname{rk}(E)} .
$$

Define stability using a strict inequality for strict nonzero subbundles. Semistability and stability are open conditions [116], and the open substack of semistable objects

$$
\mathcal{M}_{D R}^{\mathrm{se}}(r, d) \subset \mathcal{M}_{D R}(r, d)
$$

is an Artin stack of finite type (it follows from the boundedness in Nitsure's construction [116]).

Semistability of a logarithmic connection would be a consequence of semistability of the underlying bundle, but doesn't imply it in general. Esnault with Viehweg [53] and Hertling [51], and also Bolibruch [17] have studied the problem of realization of monodromy representations as logarithmic connections on 
semistable bundles in the higher genus case, generalizing Bolibruch's well-known work on $\mathbb{P}^{1}$. Our present notion of semistability of the pair $(E, \nabla)$ is somehow less subtle.

Nitsure constructs in [116] the moduli space which is a universal categorical quotient

$$
\mathcal{M}_{D R}^{\mathrm{se}}(r, d) \rightarrow M_{D R}(r, d) .
$$

The points represent $S$-equivalence classes of semistable logarithmic connections, and there is a unique polystable object in each $S$-equivalence class.

Suppose $\mathbf{c}_{1}, \ldots, \mathbf{c}_{n} \subset \mathbf{g l}(r)$ are closed subsets invariant under the adjoint action of $G L(r)$ on its Lie algebra $\mathbf{g l}(r)$. Then, as before, we obtain a closed substack

$$
\mathcal{M}_{D R}^{\mathrm{se}}\left(r, d ; \mathbf{c}_{1}, \ldots, \mathbf{c}_{n}\right) \subset \mathcal{M}_{D R}^{\mathrm{se}}(r, d)
$$

consisting of logarithmic connections $(E, \nabla)$ such that up to choice of basis of $E_{q_{i}}$, the residue $\operatorname{res}\left(\nabla, q_{i}\right)$ lies in $\mathbf{c}_{i}$.

Again, here we will concentrate on the case where each $\mathbf{c}_{i}$ is the conjugacy class of a semisimple matrix, which is closed and $G L(r)$-invariant. As above, such a conjugacy class may be parametrized by an effective divisor $g_{i} \in \operatorname{Div}\left(\mathbb{A}^{1}\right)$, with $\operatorname{deg}\left(g_{i}\right)=r$.

We denote by $\vec{g}=\left(g_{1}, \ldots, g_{n}\right)$ a vector of divisors parametrizing semisimple conjugacy classes, either $C_{i}=C\left(g_{i}\right) \subset G L(r)$ in the "multiplicative case" $g_{i} \in$ $\operatorname{Div}\left(\mathbb{G}_{m}\right)$ or $\mathbf{c}_{i}=\mathbf{c}\left(g_{i}\right) \subset \mathbf{g l}(r)$ in the "additive case" $g_{i} \in \operatorname{Div}\left(\mathbb{A}^{1}\right)$.

The rank $r$ is recovered from $\vec{g}$ as the degree of any one of the divisors $g_{i}$ (they all have to have the same degree). We can also define the trace of a divisor $g=\sum m(\alpha)[\alpha]$ to be the sum

$$
\operatorname{tr}(g):=\sum m(\alpha) \alpha \in \mathbb{C} .
$$

If $A \in \mathbf{c}(g)$ is a matrix in the corresponding conjugacy class then $\operatorname{Tr}(A)=\operatorname{tr}(g)$. The residue formula for the logarithmic connection on the determinant line bundle of $E$ provides the formula

$$
d=\operatorname{deg}(E)=\sum_{i=1}^{n} \operatorname{tr}\left(g_{i}\right) .
$$

This obviously has to be an integer, otherwise the moduli space will be empty. The degree $d$ of the bundle $E$ may be recovered from $\vec{g}$. Thus, we are justified 
in writing

$$
M_{D R}(\vec{g}):=M_{D R}\left(r, d ; \mathbf{c}\left(g_{1}\right), \ldots, \mathbf{c}\left(g_{n}\right)\right)
$$

2.6. Deformations and obstructions. Suppose $(E, \nabla)$ represents a point in $M_{D R}(\vec{g})$. We compare the deformation and obstruction theory of $(E, \nabla)$ as logarithmic connection, with that of $(E, \nabla)$ as a point in the moduli stack $\mathcal{M}_{D R}(\vec{g})$. The following result was pointed out by N. Katz in the late 1980's.

Theorem 2.9. The deformation and obstruction theory for the moduli stack $\mathcal{M}_{D R}(\vec{g})$ at a point $(E, \nabla)$ is governed by the middle cohomology groups of the endomorphism bundle $\mathbb{H}^{i} M D R(Y, E n d(E))$ for $i=0$ (automorphisms), $i=1$ (deformations) and $i=2$ (obstructions).

Proof: We give an heuristic but ultramodern explanation. Definitions and explicitations would need to be filled in, but this should convince the reader why it is true.

The deformation and obstruction theory of $(E, \nabla)$ as a logarithmic connection, that is as a point in $\mathcal{M}_{D R}(r, d)$, is given by the $L_{\infty}$ algebra

$$
\mathbf{D}_{E, \nabla}\left(\mathcal{M}_{D R}\right):=\mathbb{H}^{\cdot}\left(Y, \operatorname{End}(E) \otimes \Omega_{Y}(\log Q)\right) .
$$

Let $R:=\operatorname{gl}(r) / / G L(r)$ denote the moduli stack of conjugacy classes of matrices. It is a smooth Artin algebraic stack. At a point corresponding to a matrix $A$, its deformation theory is controlled by the $L_{\infty}$-algebra concentrated in degrees 0 and 1

$$
\mathbf{D}_{A}(R):=\mathbf{g l}(r) \stackrel{[-, A]}{\longrightarrow} \mathbf{g l}(r) .
$$

If $A$ is in the semisimple conjugacy class $\mathbf{c}_{i}$, then the deformation theory of $\left\langle\mathbf{c}_{i}\right\rangle:=\mathbf{c}_{i} / / G L(r)$ is controlled by

$$
\begin{aligned}
\mathbf{D}_{A}\left(\left\langle\mathbf{c}_{i}\right\rangle\right) & =[\operatorname{gl}(r) \stackrel{[-, A]}{\longrightarrow}(\operatorname{Im}(u \mapsto[u, A]))] \\
= & \operatorname{ker}\left(\mathbf{D}_{A}(R) \rightarrow \operatorname{gl}(r)^{A}\right)
\end{aligned}
$$

where $\mathbf{g l}(r)^{A}$ is the space of cofixed or vectors of the adjoint action of $A$, which is isomorphic to the space of fixed vectors since $A$ is semisimple. In practical terms, if $A$ is in diagonal form then the degree one piece of $\mathbf{D}_{A}\left(\left\langle\mathbf{c}_{i}\right\rangle\right)$ is the space of off-block-diagonal matrices (for the blocks determined by the eigenvalues of $A$ ) and $\mathbf{g l}(r)^{A}$ is the space of block-diagonal matrices. 
For any point $q_{i} \in Q$ the construction $(E, \nabla) \mapsto \operatorname{res}\left(\nabla, q_{i}\right)$ gives a morphism of moduli stacks $\mathcal{M}_{D R}(r, d) \rightarrow R$. Putting these together gives a morphism $\mathcal{M}_{D R}(r, d) \rightarrow R^{n}$. On the other hand, a vector of divisors $\vec{g}$ represents a collection of conjugacy classes $\mathbf{c}\left(g_{i}\right)$ which gives the substack

$$
R(\vec{g}):=\prod_{i=1}^{n}\left\langle\mathbf{c}\left(g_{i}\right)\right\rangle \subset R^{n},
$$

and by definition

$$
\mathcal{M}_{D R}(\vec{g})=\mathcal{M}_{D R}(r, d) \times_{R^{n}} R(\vec{g}) .
$$

To get the deformation theory for this fiber product, we should take the homotopy fiber product of the $L_{\infty}$-algebras. The one for $R(\vec{g})$ is the kernel of a map (2.2) on the one for $R^{n}$.

Hinich explains how to go between a sheaf of $L_{\infty}$-algebras, and a global $L_{\infty^{-}}$ algebra [68]. In our case, $\mathbf{D}_{E, \nabla}\left(\mathcal{M}_{D R}\right)$ is the globalization of the sheaf of $L_{\infty^{-}}$ algebras $D R(Y, \operatorname{End}(E)$ ) (the logarithmic de Rham complex along $Q$ ). Going back and forth a few times we see that the deformation theory for $\mathcal{M}_{D R}(\vec{g})$ is controlled by the globalization, or hypercohomology, of the kernel of the map corresponding to (2.2) on the sheaf of $L_{\infty}$-algebras, this map expressed in local terms at the singularities as

$$
D R(Y, \operatorname{End}(E)) \rightarrow \bigoplus_{i=1}^{n} \operatorname{End}(E)_{q_{i}}^{0}
$$

where the superscript 0 means the trivial eigenspace for the action of $\operatorname{res}\left(\nabla, q_{i}\right)$. The kernel is exactly the middle de Rham complex for $\operatorname{End}(E)$. Thus, the deformation theory is controlled by an $L_{\infty}$-algebra $\mathbb{H} \cdot M D R(Y, \operatorname{End}(E))$.

Corollary 2.10. Suppose $(E, \nabla) \in \mathcal{M}_{D R}(\vec{g})$ is a point corresponding to an irreducible representation. Then it is a smooth point of the moduli stack, and has only scalar automorphisms so it is also a smooth point of the moduli space where the tangent space is $\mathbb{H}^{1} M D R(Y$, End $(E))$.

Proof: Decompose into the trace-free part and the scalars: $\operatorname{End}(E)=E n d^{\prime}(E) \oplus$ $\mathcal{O}$. The trace of the obstruction map is zero, and $\mathbb{H}^{1} M D R(Y, \mathcal{O})=0$ since $Y \cong$ $\mathbb{P}^{1}$. Thus, the deformations and obstructions are given by $\mathbb{H}^{1} M D R\left(Y, E n d^{\prime}(E)\right)$ and $\mathbb{H}^{2} M D R\left(Y, E n d^{\prime}(E)\right)$ respectively. Poincaré duality for the middle cohomology, plus the fact that $E n d^{\prime}(E)$ is self-dual, gives $\mathbb{H}^{2} M D R\left(Y, E n d^{\prime}(E)\right) \cong$ 
$\mathbb{H}^{0} M D R\left(Y, E n d^{\prime}(E)\right)=0$ since $E$ has no trace-free endomorphisms because it is irreducible. Thus the space of obstructions vanishes, and the tangent space is given by $\mathbb{H}^{1} M D R(Y, \operatorname{End}(E))=\mathbb{H}^{1} M D R\left(Y, \operatorname{End}^{\prime}(E)\right)$.

2.7. Dimension counting. From the previous discussion of deformations and obstructions, we find the following boiled-down statement.

Proposition 2.11. Suppose $(E, \nabla)$ is a stable point in $M_{D R}^{s}(\vec{g})$. Then the dimension of the moduli space at the given point (or any other stable point) is obtained by a naive dimension count:

$$
\operatorname{dim}\left(M_{D R}^{s}(\vec{g})\right)=\sum_{i=1}^{n} \operatorname{dim}\left(\mathbf{c}\left(g_{i}\right)\right)-2 r^{2}+2 .
$$

The factor $2\left(r^{2}-1\right)$ corresponds to the fact that the conjugation action factors through $P G L(r)$ and the product identity lies in $S L(r)$.

The same dimension count holds for the open subset of irreducible representations $M_{B}^{\mathrm{irr}}(\vec{g})$ if it is nonempty.

Proof: Apply Corollary 2.10. The tangent space is $\mathbb{H}^{1} M D R(Y, \operatorname{End}(E))$ and

$$
\mathbb{H}^{0} M D R(Y, \operatorname{End}(E))=\mathbb{H}^{2} M D R(Y, \operatorname{End}(E))=\mathbb{C}
$$

since $E$ is irreducible. Obtain the dimension count by using the fact that $M D R(Y$, $\operatorname{End}(E))$ is the kernel of the map (2.3), noting

$$
\operatorname{dim}\left(\mathbf{c}\left(g_{i}\right)\right)=r^{2}-\operatorname{dim} E n d(E)_{q_{i}}^{0},
$$

and calculating the Euler characteristic. The Riemann-Hilbert correspondence gives the corresponding statement for $M_{B}^{\mathrm{irr}}(\vec{g})$.

We introduce the defect. It may be seen as playing a role in the dimension count, but is also foremost related to Katz's algorithm as we shall explain later. For each $g_{i}$, let $\nu\left(g_{i}\right)$ be the maximal multiplicity of an eigenvalue. The centralizer of a matrix $A$ in the conjugacy class $\mathbf{c}\left(g_{i}\right)$ is the set of block-diagonal matrices, and the dimension of the conjugacy class is the number of positions which are not in the block-diagonal pieces.

Think visually of shifting all of the diagonal blocks to the left of the matrix. In other words, transpose each square diagonal block with the rectangle consisting of all places to the left of it in the same rows. In the resulting picture, the square 
blocks are now arrayed from top to bottom flush with the left edge of the matrix. The dimension of the conjugacy class is still the number of positions which are not in these blocks. This leftover part can be divided into two regions: a big rectangle of size $r \times\left(r-\nu\left(g_{i}\right)\right)$ which is everything to the right of the biggest block, plus a union of other rectangular regions corresponding in each row to the positions to the right of the edge of the corresponding block for that row, but to the left of the size of the biggest block. The second piece might be empty, indeed it is empty exactly in the case when the blocks all have the same size. We obtain the crude estimate

$$
\operatorname{dim}\left(\mathbf{c}\left(g_{i}\right)\right) \geq r\left(r-\nu\left(g_{i}\right)\right),
$$

leading to the crude estimate for the dimension of the moduli space as

$$
\operatorname{dim}\left(M_{D R}^{s}(\vec{g})\right) \geq n r^{2}-r \sum_{i=1}^{n} \nu\left(g_{i}\right)-2 r^{2}+2=2+r\left((n-2) r-\sum_{i=1}^{n} \nu\left(g_{i}\right)\right) .
$$

In view of this formula already, it seems reasonable to consider the quantity

$$
\delta(\vec{g}):=(n-2) r-\sum_{i=1}^{n} \nu\left(g_{i}\right) .
$$

We call this the defect because it enters into Katz's algorithm in a remarkably elegant way: if $\beta$ is a convolution object corresponding to a choice of maximalmultiplicity eigenvalue for each $g_{i}$, then the new rank of the Katz-transformed local monodromy data is

$$
r^{\prime}=r+\delta(\vec{g})
$$

We will want to run Katz's algorithm when $\delta(\vec{g})$ is negative. We can do so until we get to a vector whose defect is positive.

In terms of the defect, the crude dimension count says $\operatorname{dim}\left(M_{D R}^{s}(\vec{g})\right) \geq 2+$ $r \delta(\vec{g})$. In order to refine the dimension count, introduce the superdefect denoted locally by

$$
\sigma\left(g_{i}\right):=\operatorname{dim}\left(\mathbf{c}\left(g_{i}\right)\right)-r\left(r-\eta\left(g_{i}\right)\right),
$$

and globally by

$$
\sigma(\vec{g}):=\sum_{i=1}^{n} \sigma\left(g_{i}\right)
$$

These quantities, which are always $\geq 0$, are just the differences between the crude dimension counts and the actual dimensions. Thus we have, when the stable open 
set is nonempty,

$$
\operatorname{dim}\left(M_{D R}^{s}(\vec{g})\right)=2+r \delta(\vec{g})+\sigma(\vec{g}) .
$$

In view of the possibility of applying Katz's algorithm to decrease the rank whenever $\delta<0$, the remaining case to investigate is when $\delta(\vec{g}) \geq 0$. Under this hypothesis, the dimension of the moduli space is always $\geq 2$. The cases of dimension 0 were the subject of Katz's original book: they arise when one ends up with a rank one local system, at which point it will no longer be possible to find a convoluter satisfying Convention 3.1. The case of dimension 2 is particularly interesting, although unfortunately our construction of $\S 6$ (Corollary 6.10) will not apply.

Lemma 2.12. The superdefect $\sigma\left(g_{i}\right)$ vanishes if and only if all of the eigenvalues of $g_{i}$ have the same multiplicity. In the domain $\delta(\vec{g}) \geq 0$, the dimension of the moduli space will be exactly 2 , if and only if $\delta=0$ and the superdefects $\sigma\left(g_{i}\right)$ all vanish.

Proof: Note that $\sigma\left(g_{i}\right)$ is the number of places left over in the complement of the pushed-left diagonal blocks, after taking out the big rectangle. This vanishes only if all of the blocks have the same size. The last statement follows from $\sigma(\vec{g}) \geq 0$.

If $\vec{g}$ is an example of the case $\delta=\sigma=0$ then any multiple (meaning to multiply all of the divisors $g_{i}$ by the same amount) is also an example. Thus the examples of this case come in families which are indexed by an integer $d \geq 0$. Following Kostov, write the type of $g_{i}$ as a partition of $r$, for example $(d, d)$ indicates a divisor of the form $d[a]+d[b]$ supported at two eigenvalues $a$ and $b$ both with multiplicity $d$. Then $\vec{g}$ has type given by a "polymultiplicity vector" $P M V(\vec{g})$ which is a vector of partitions. Applying the definition of the defect we immediately see the following, due to Kostov in the paper [90] where he investigates explicitly the resulting list of cases:

Lemma 2.13. In the domain $\delta(\vec{g}) \geq 0$, the only cases where $\operatorname{dim}\left(M_{D R}^{s}(\vec{g})\right)=2$ are the following four:

$$
\begin{aligned}
& \text {-for } n=4, d=r / 2 \text { and } \operatorname{PMV}(\vec{g})=((d, d),(d, d),(d, d),(d, d)) ; \\
& \text {-for } n=3, d=r / 3 \text { and } \operatorname{PMV}(\vec{g})=((d, d, d),(d, d, d),(d, d, d)) ; \\
& \text {-for } n=3, d=r / 4 \text { and } \operatorname{PMV}(\vec{g})=((2 d, 2 d),(d, d, d, d),(d, d, d, d)) ; \text { and } \\
& \text {-for } n=3, d=r / 6 \text { and } \operatorname{PMV}(\vec{g})=((3 d, 3 d),(2 d, 2 d, 2 d),(d, d, d, d, d, d)) .
\end{aligned}
$$


Proof: Apply Lemma 2.12. Vanishing of the superdefect means that $g_{i}$ is of type $\left(d_{i}, \ldots, d_{i}\right)$ for some $d_{i}=r / b_{i}$. Vanishing of the defect says $\sum_{i}\left(1 / b_{i}\right)=n-2$, and the only solutions with $b_{i}$ integers $\geq 2$ are

$$
2=\frac{1}{2}+\frac{1}{2}+\frac{1}{2}+\frac{1}{2}, 1=\frac{1}{3}+\frac{1}{3}+\frac{1}{3}, 1=\frac{1}{2}+\frac{1}{4}+\frac{1}{4}, 1=\frac{1}{2}+\frac{1}{3}+\frac{1}{6} .
$$

These give the cases of the lemma.

Exercise 2. Classify the possible polymultiplicity vectors for $\bar{g}$ in the cases when the moduli space has dimension 4 and 6 .

\section{The DiAgONAL CONFIGURATION AND ITS BLOWING UP}

The convolution operation comes from the diagonal configuration consisting of vertical and horizontal lines plus the diagonal. This kind of configuration is a recurring theme in Hirzebruch's work [69].

In what follows, put $Y:=\mathbb{P}^{1}$ and $Z:=\mathbb{P}^{1}$, and look at the product $Z \times Y$. We have the projections $\xi: Z \times Y \rightarrow Z$ and $\eta: Z \times Y \rightarrow Y$.

Fix a subset of distinct points $Q:=\left\{q_{1}, \ldots, q_{n}\right\} \subset \mathbb{P}^{1}$ and let $Q_{Y}$ or $Q_{Z}$ denote this subset considered as a divisor in $Y$ or $Z$ respectively. Let

$$
D:=Z \times Q_{Y} \cup Z \times Q_{Z} \times Y \cup \Delta \subset Z \times Y \text {. }
$$

be the divisor obtained by using the divisors $Q_{Z}$ and $Q_{Y}$ in the vertical and horizontal directions, and adding the diagonal. Denote also by $\eta$ and $\xi$ the projections

$$
\xi:(Z \times Y)-D \longrightarrow Z-Q_{Z}, \quad \eta:(Z \times Y)-D \longrightarrow Y-Q_{Y} .
$$

The pair $(Z \times Y, D)$ is the diagonal configuration.

The divisor $D$ does not have normal crossings, indeed the diagonal meets the other components in a series of triple points. In order to obtain a variety with normal crossings compactification compatible with the projection maps, we have to blow up the diagonal configuration at these triple crossing points . Let $X$ be the resulting variety, thus we have a birational map

$$
X \rightarrow Y \times Z
$$


obtained by blowing up the points $\left(q_{1}, q_{1}\right), \ldots,\left(q_{n}, q_{n}\right)$. Let $J \subset X$ denote the reduced inverse image of the divisor $D$. We have a decomposition

$$
J=T+U_{1}+\cdots+U_{n}+H_{1}+\cdots+H_{n}+V_{1}+\cdots+V_{n},
$$

where:

$-T$ is the strict transform of the diagonal $\Delta$;

$-H_{i}$ is the horizontal strict transform of $Z \times\left\{q_{i}\right\}$;

$-V_{i}$ is the vertical strict transform of $\left\{q_{i}\right\} \times Y$; and

$-U_{i}$ is the exceptional divisor lying over $\left(q_{i}, q_{i}\right)$.

These intersect as follows: each $U_{i}$ meets $T, H_{i}$ and $V_{i}$ in three distinct points. Also $H_{i}$ meets $V_{j}$ for $i \neq j$. These intersections are transverse, and there are no other intersections.

Let $\xi: X \rightarrow Z$ denote the first projection. It is seen as going in the vertical direction, so it contracts $U_{i}+V_{i}$ to the point $q_{i}$ and indeed $\xi^{-1}\left(q_{i}\right)=U_{i}+V_{i}$. Let $\eta: X \rightarrow Y$ denote the second projection going in the horizontal direction, so $\eta^{-1}\left(q_{i}\right)=U_{i}+H_{i}$.

The other divisor components are mapped isomorphically onto the bases of these projections:

$$
\xi: T \stackrel{\cong}{\rightrightarrows} Z, \quad \xi: H_{i} \stackrel{\cong}{\rightrightarrows} Z
$$

and

$$
\eta: T \stackrel{\cong}{\rightarrow} Y, \quad \eta: V_{i} \cong
$$

These divisor components intersect transversally all fibers of $\xi$ or $\eta$ respectively.

3.1. Convoluters - the Betti version. The basic setup of Katz's convolution operation is to take a local system on $Y$, pull it back to $Y \times Z$ or the blowup $X$, tensor with a rank one local system, and push forward to $Z$ using $\mathbb{R}^{1} \xi_{*}$. Obviously, the first step in understanding and calculating this, is to understand the rank one objects [25] [56] over $(X, J)$. We look at the "Betti" case of local systems or representations of the fundamental group.

The birational blowing-up morphism is an isomorphism outside $J$ and $D$, that is

$$
X-J \stackrel{\cong}{\rightrightarrows}(Z \times Y)-D .
$$

In particular, local systems on one or the other are the same thing. We denote generically by $\beta$ our convoluter, in this case a local system. It is given by a 
representation of the fundamental group into $\mathbb{C}^{*}$, a representation which factors through the abelianization as

$$
\beta: H_{1}(X-J, \mathbb{Z}) \rightarrow \mathbb{C}^{*}
$$

The loops $\gamma_{H_{i}}, \gamma_{V_{i}}$ and $\gamma_{T}$ going around the respectively denoted components of $J$, generate the first homology of $H_{1}(X-J)$. For our calculations it is convenient to include $\gamma_{U_{i}}$ also as generators. These are subject to the following relations:

$$
\gamma_{T}+\sum_{i=1}^{n} \gamma_{H_{i}}=0, \quad \gamma_{T}+\sum_{i=1}^{n} \gamma_{V_{i}}=0, \quad \gamma_{U_{i}}=\gamma_{T}+\gamma_{H_{i}}+\gamma_{V_{i}}(i=1, \ldots, n),
$$

coming respectively from a vertical $\mathbb{P}^{1}$ intersecting $T$ and the $H_{i}$; from a horizontal $\mathbb{P}^{1}$ intersecting $T$ and the $V_{i}$; and for $i=1, \ldots, n$ from a small $\mathcal{C}^{\infty}$ deformation of the exceptional $U_{i}$, which meets $T, H_{i}$ and $V_{i}$ and intersects the undeformed $U_{i}$ negatively. That these generate the module of relations, can be seen from a Leray spectral sequence argument.

Denote by $\beta^{H_{i}}$ the monodromy of $\beta$ on the loop $\gamma_{H_{i}}$ and similarly for the other generators. Thus, specifying a local system of rank one on $X-J$ comes down to specifying

$$
\beta^{H_{i}}, \beta^{V_{i}}, \beta^{U_{i}}, \beta^{T} \in \mathbb{C}^{*}
$$

subject to the relations

$$
\beta^{T} \cdot \prod_{i=1}^{n} \beta^{H_{i}}=1, \quad \beta^{T} \cdot \prod_{i=1}^{n} \beta^{V_{i}}=1, \quad \beta^{U_{i}}=\beta^{T} \cdot \beta^{H_{i}} \cdot \beta^{V_{i}} .
$$

Of course the last relations mean that $\beta^{U_{i}}$ are redundant.

We will use our convoluters to define a convolution operation, in which the diagonal $\Delta$ plays a primordial role. It will be important to have nontrivial monodromy around the diagonal. To simplify notation set

$$
\chi:=\beta^{T}
$$

and make the following convention.

Convention 3.1. The monodromy around the diagonal of our convoluter is nontrivial, that is $\chi \neq 1$. 
3.2. Convoluters - the de Rham version. We will find it most convenient to restrict to convolution with rank one logarithmic connections on the trivial bundle, that is de Rham objects of the form $\left(\mathcal{O}_{X}, d+\beta\right)$ where $\beta$ is a one form on $X$ with logarithmic poles along $J$. The more general case can be viewed as being subsumed by the theory of parabolic logarithmic $\lambda$-connections [112], see $\S 7$.

In the present case, then, a convoluter is just a section

$$
\beta \in H^{0}\left(X, \Omega_{X}^{1}(\log J)\right) .
$$

Given $\beta$ we denote by $\beta^{H_{i}}, \beta^{V_{i}}, \beta^{U_{i}}$ and $\beta^{T}$ its residues along $H_{i}, V_{i}, U_{i}$ and $T$ respectively. Note that $H^{1}\left(X, \mathcal{O}_{X}\right)=0$, so by Deligne's mixed Hodge theory

$$
\int: H^{0}\left(X, \Omega_{X}^{1}(\log J)\right) \cong H^{1}\left((X-J)^{\text {top }}, \mathbb{C}\right)
$$

and the integrals over $\gamma_{H_{i}}$ etc. are $2 \pi \sqrt{-1}$ times the residues $\beta^{H_{i}}$ etc. In particular, the structure of $H_{1}(X-J, \mathbb{Z})$ recalled in the previous subsection implies that $\beta$ is determined by its residues, and these are subject to the equations

$$
\beta^{T}+\sum_{i=1}^{n} \beta^{H_{i}}=0, \quad \beta^{T}+\sum_{i=1}^{n} \beta^{V_{i}}=0, \quad \beta^{U_{i}}=\beta^{T}+\beta^{H_{i}}+\beta^{V_{i}} .
$$

The de Rham convolution object $\beta$ gives rise to a local system, or Betti convoluter $\varphi$ with

$$
\varphi^{H_{i}}=e^{2 \pi \sqrt{-1} \beta^{H_{i}}}, \ldots
$$

The analogue of Convention 3.1 is:

Convention 3.2. The residue $\beta^{T}$ of $\beta$ along the diagonal is not an integer.

\section{Middle Convolution-Betti Version}

In this section we will work with the divisor $D \subset(Z \times Y)$, and do our computations in braid-group style [113] [132] [45]. One could alternatively use the blowing up $(Z, J)$ and give a treatment similar to the one we will give in the de Rham case later.

The discussion of this section is the complex geometric version of Katz's construction. Katz gave a geometric definition of middle convolution in [86, 2.7-2.9]. His formulae there, stated in the context of perverse $\ell$-adic sheaves, work universally in any geometric context. The complex geometric version was defined 
more explicitly, and first exploited by Dettweiler and Reiter [44] [45] [46], and Völklein, Strambach, [139] [132]. They write down explicit matrices but the motivation comes from braid-style computations. Kostov proposes an ingenious version of the construction which doesn't refer to the geometric picture, but instead is based on the possibility of multiplying the connection matrix by a scalar to get to the case of integer eigenvalues [91]. And, Crawley-Boevey views the construction, again in algebraic terms, as something about root systems. Boalch considered a particular example of middle convolution in a non-rigid case [15], and the link with Katz's construction was made in [47]. In [45] following [86, Chap. 5.1] it is shown that the explicit matrix definition of $M C$ has a geometric or cohomological interpretation as a higher direct image - this is the point of view we adopt here. The braid-style calculations of group cohomology necessary to get the local form of monodromy out of this geometric definition were done in [45] but using the Pochammer basis for the group cohomology classes, rather than a standard basis as we shall use here. In spite of the numerous references on this subject, we go through the details, where possible keeping simplifying assumptions for our expository purpose.

\subsection{Definitions. Recall that}

$$
\eta: Z \times Y \rightarrow Y, \quad \xi: Z \times Y \rightarrow Z
$$

are the projections, and use the same name for the projections on the open subset $(Z \times Y)-D$. A convoluter $\beta$ is a rank one local system on $(Z \times Y)-D$.

Define the raw convolution $R C_{\beta}$ as follows. If $L$ is a local system on $Y-Q_{Y}$ then put

$$
R C_{\beta}(L):=R^{1} \xi_{*}\left(\beta \otimes\left(\eta^{*} L\right)\right) .
$$

It is a local system on $Z-A_{Z}$. The middle convolution $M C_{\beta}(L)$ will be a subsystem of $R C_{\beta}(L)$ the kernel of the map to some natural essentially local systems coming from triviality of certain pieces of the local monodromy transformations. It corresponds to replacing the cohomology of the fibers in the $R^{1} \xi_{*}$ construction, by the middle cohomology discussed in $\S 2.1$ above.

In order to make explicit calculations, we will adopt the viewpoint of homology rather than cohomology. Let $R C_{\beta}^{*}(L)$ denote the local system obtained by taking the homology of the fibers with coefficients in $\beta \otimes \eta^{*} L$. Let $M C_{\beta}^{*}(L)$ denote the quotient corresponding to "middle homology" defined by duality with middle 
cohomology (the precise definition will appear in our discussion below). If we let $\beta^{*}$ and $L^{*}$ denote the dual local systems, then by the duality between homology and cohomology we have

$$
R C_{\beta}^{*}(L)=\left(R C_{\beta^{*}}\left(L^{*}\right)\right)^{*}
$$

and similarly for $M C^{*}$. Thus it is equivalent if we look at homology, and it is easier to visualize geometrically classes in homology with local coefficients.

4.2. Computations in group homology. We would like to calculate the local monodromy transformations of the raw and then middle convolutions. In order to do this, we transform the question into a computation of the action of the fundamental group of the base, on the group homology of the fiber. See [139] [45] [42] for example.

In order to speak of fundamental groups, we need to choose basepoints. Choose a basepoint $b \in Y-Q$. For $z \in Z-Q_{Z}$ the fiber of $X-J$ over $z$ is $Y-Q-\{z\}$. This has $(z, b)$ as basepoint whenever $z \neq b$. In particular, in order to get a fibration of based spaces we should additionally take the point $b$ out of the base. For this reason, put $Q_{Z}^{b}:=Q_{z} \cup\{b\}$.

On the other hand, we would like to consider the fundamental group of $Z-Q_{Z}^{b}$. Choose another basepoint $c \in Z-Q_{Z}^{b} \subset \mathbb{P}^{1} \cong Y$. In the fiber over $c \in Z$ we have the complement of $Q_{Y}$ and should also take out the diagonal point $(c, c)$. Thus, let $Q_{Y}^{c}:=Q_{Y} \cup\{c\}$.

The fiber $Y_{c}=\{c\} \times\left(Y-Q_{Y}^{c}\right)$ of the projection $\xi:(X-J) \rightarrow\left(Z-Q_{Z}^{b}\right)$ over $c$, is an open Riemann surface pointed by the basepoint $(c, b)$.

Let $\left.\Gamma:=\pi_{1}\left(Y_{c},(c, b)\right)\right) \cong \pi_{1}\left(Y-Q_{Y}^{c}, b\right)$. It is a free group. The fundamental group of the base $\Upsilon:=\pi_{1}\left(Z-Q_{Z}^{b}, c\right)$ acts on $\Gamma$. We will describe the action in greater detail below. Denote the action by $u \mapsto(\gamma \mapsto \mu(u, \gamma))$ for $u \in \Upsilon, \gamma \in \Gamma$.

Make the convention for group composition that $a b$ means $b$ followed by $a$. That way, a monodromy representation indicates transport of sections along the path and satisfies $\rho(a b)=\rho(a) \rho(b)$.

The local system $R C_{\beta}^{*}(L)$ restricted to $Z-Q_{Z}^{b}$ can be described as follows. The local system $L$ corresponds to a representation $\rho$ of $\Gamma$ on the vector space 
$L_{b}$, invariant with respect to the action of $\Upsilon$ in the sense that

$$
\rho(\mu(u, \gamma))=\rho(\gamma)
$$

for any $\gamma \in \Gamma$ and $u \in \Upsilon$. Similarly, the local system $\beta$ corresponds to a pair of characters

$$
\beta^{c, \cdot}: \Gamma \rightarrow \mathbb{C}^{*}, \quad \beta^{, b}: \Upsilon \rightarrow \mathbb{C}^{*}
$$

and the first of these is again invariant, $\beta^{c, \cdot}(\mu(u, \gamma))=\beta^{c, \cdot}(\gamma)$. Tensoring together we obtain a representation denoted

$$
\rho^{\beta}: \gamma \mapsto \beta^{c, \cdot}(\gamma) \rho(\gamma)
$$

again invariant with respect to the action of $\Upsilon$. The local system $R C_{\beta}(L)$ (resp. $\left.R C_{\beta}^{*}(L)\right)$ corresponds to the vector space $H^{1}\left(\Gamma, \rho^{\beta}\right)\left(\right.$ resp. $\left.H_{1}\left(\Gamma, \rho^{\beta}\right)\right)$. The action of $\Upsilon$ is obtained by the natural action, tensored with the character $\beta^{\cdot, b}$. This tensorization is due to the fact that the local system $\beta$ is not trivial on the basepoint section $z \mapsto(z, b)$ over $Z-Q_{Z}^{b}$.

Denote by $V$ the vector space $\mathbb{C}^{r}$ on which the representation $\rho^{\beta}$ is defined. Denote by $H^{1}(\Gamma, V)$ the cohomology and $H_{1}(\Gamma, V)$ the homology.

Fix generators for $\Gamma$ as follows: we have loops $\alpha_{1}, \ldots, \alpha_{n}$ going clockwise around the points $q_{1}, \ldots, q_{n}$ in the standard way, and $\delta$ going clockwise around the point $(c, c) \in \Delta$ in the fiber $\{c\} \times\left(Y-Q_{Y}^{c}\right)$. The group $\Gamma$ has generators $\alpha_{i}, \delta$ subject to the single relation

$$
\delta \alpha_{1} \cdots \alpha_{n}=1 .
$$

Using this relation any one of the generators could be ignored but it will be more convenient to keep all of them.

The character $\beta^{c, \cdot}$ acts on these generators as follows: $\beta^{c, \cdot}\left(\alpha_{i}\right)=\beta^{V_{i}}, \quad \beta^{c, \cdot}(\delta)=$ $\beta^{T}$. Thus we have

$$
\rho^{\beta}(\delta)=\beta^{T} \cdot 1^{r}, \quad \rho^{\beta}\left(\alpha_{i}\right)=\beta^{V_{i}} \rho\left(\alpha_{i}\right), \quad i=1, \ldots, n .
$$

The homology $H_{1}(\Gamma, V)$ is the homology at degree one of the sequence

$$
C_{2}(\Gamma, V) \rightarrow C_{1}(\Gamma, V) \rightarrow C_{0}(\Gamma, V)=V .
$$

Furthermore, $C_{1}(\Gamma, V)$ is the $\mathbb{C}$-vector space formally generated by the symbols $G(\gamma, v)$ where $\gamma \in \Gamma$ and $v \in V$, subject only to the relation of $\mathbb{C}$-linearity in the variable $v$. And $C_{2}(\Gamma, V)$ is generated by symbols $Q(\gamma, \xi, v)$ where $\gamma, \xi \in \Gamma$ and 
$v \in V$. Geometrically, $G(\gamma, v)$ represents a cycle which starts with value $v$ and continues along the path $\gamma$. And $Q(\gamma, \xi, v)$ represents a simplex whose sides are $\gamma, \xi$ and $\xi \gamma$ with coefficient $v$ at the starting point.

The boundary operators for the complex are $\partial G(\gamma, v)=\gamma(v)-v, \quad \partial Q(\gamma, \xi, v)=G(\gamma, v)+G(\xi, \gamma(v))-G(\xi \gamma, v), \quad \partial \circ \partial=0$.

We work with the vector space $C_{1}(\Gamma, V) / \partial C_{2}(\Gamma, V)$ denoted just $C_{1} / \partial C_{2}$ for short. It is finite dimensional, and its elements are $\mathbb{C}$-linear combinations of classes denoted $G[\gamma, v]$ which are now subject to the relations that this symbol is $\mathbb{C}$-linear in $v$, and that

$$
G[\xi \gamma, v]=G[\gamma, v]+G[\xi, \gamma(v)] .
$$

If we fix a basis $\left\{v_{j}\right\}$ for $V$ then from the set of generators of $\Gamma$ we obtain a basis for $C_{1} / \partial C_{2}$ consisting of the $G\left[\alpha_{i}, v_{j}\right]$ and $G\left[\delta, v_{j}\right]$. It will be useful in what follows to have a formula for multiple products. For example

$$
G[\eta \xi \gamma, v]=G[\gamma, v]+G[\xi, \gamma(v)]+G[\eta, \xi \gamma(v)]
$$

and more generally $G\left[\gamma_{1} \cdots \gamma_{m}, v\right]=\sum_{i=1}^{m} G\left[\gamma_{i}, \gamma_{i+1} \cdots \gamma_{m}(v)\right]$. Similarly for the inverse, the equation

$$
0=G\left[\gamma \gamma^{-1}, v\right]=G\left[\gamma^{-1}, v\right]+G\left[\gamma, \gamma^{-1}(v)\right]
$$

gives $G\left[\gamma^{-1}, v\right]=-G\left[\gamma, \gamma^{-1}(v)\right]$.

Now consider the action of $\Upsilon$. What we call the "natural action" is the one coming from the action on the explicit generators written above. This corresponds to tensoring to trivialize the restriction of the local system on the basepoint section. In the end, since the local system is not trivial on the basepoint section, we will have to take the natural action tensored with the character $\beta^{,}, b$.

The generator $u_{i}$ of $\Upsilon$ corresponds to a path where the point $c$ goes around the point $q_{i}$. There is some choice about how to arrange this picture, with respect to the picture of the standard generators of $\Gamma$. Think of the points $q_{1}, \ldots, q_{n}$ as lined up in a row, with the basepoint $b$ off to one side so that the points are arrayed from left to right when viewed from $b$. The paths $\alpha_{i}$ go straight from $b$ to $q_{i}$, once around clockwise, then back to $b$. On the other hand, let $c$ be on the other side of the row of points $q_{i}$. We obtain a number of paths $\delta=\delta_{1}, \ldots, \delta_{n}$ going from $b$ to $c$, around clockwise, and back to $b$. These are defined by saying 
that the starting and ending path for $\delta_{i}$ goes just to the left of the point $q_{i}$, for $1<i \leq n$ the path goes between $q_{i-1}$ and $q_{i}$. We have the relation

$$
\delta_{i+1}=\alpha_{i} \delta_{i} \alpha_{i}^{-1} \text {. }
$$

In particular the $\delta_{i}$ are all conjugate to $\delta=\delta_{1}$, which implies that $\rho^{\beta}\left(\delta_{i}\right)$ are always multiplication by $\beta^{T}$.

Now, define $u_{k}$ as the path which sends $c$ straight to $q_{k}$, around clockwise, and back to its starting point. This happens on the other side of our picture from the paths starting at $b$.

With this picture, the action of $u_{k}$ doesn't change the $\alpha_{j}$ for $j \neq k$. On the other hand, we have a Dehn twist between $\delta_{k}$ and $\alpha_{k}$. Notice that the introduction of the different conjugates $\delta_{k}$ allows us to represent these Dehn twists uniformly for each $k$; if we try to write down the formula with $\delta=\delta_{1}$ it becomes more complicated.

A geometric look at the picture of $c$ going clockwise around $q_{k}$ yields:

Proposition 4.1. The action of $u_{k}$ on $\Gamma$ is given by

$$
\mu\left(u_{k}, \alpha_{k}\right)=\delta_{k}^{-1} \alpha_{k} \delta_{k}
$$

and

$$
\mu\left(u_{k}, \delta_{k}\right)=\delta_{k}^{-1} \alpha_{k}^{-1} \delta_{k} \alpha_{k} \delta_{k}
$$

Exercise 3. Define conjugates $\zeta_{i}$ where the point $c$ goes in between $q_{i-1}$ and $q_{i}$, around $b$, and back. Describe the action of $\zeta_{i}$. We have the relations $u_{1} \cdots u_{i-1}$ $\zeta_{i} u_{i} \cdots u_{n}=1$ in $\Upsilon$. Check that the action defined by the above formulae for the $u_{i}$ plus the formulae for $\zeta_{i}$, satisfies these relations.

4.3. The local monodromy transformations. Now we would like to compute the eigenvalues of the monodromy transformations. This computation is local around one of the points $q_{k}$. For our present purposes we only ask for the Jordan normal form of the monodromy transformation. More precise information, in fact the explicit monodromy matrices with respect to the Pochammer basis, are obtained in [45], [42, Lemma 3.3.5, Proposition 3.3.6]. Our computation is along these lines but we don't need to consider the Pochammer elements. 
To reduce notation put

$$
\chi:=\beta^{T}, \quad \beta_{i}:=\beta^{H_{i}} .
$$

The values of $\beta^{V_{i}}$ don't matter, as we are initially calculating the natural action trivialized over the basepoint section. Our previous formulae become

$$
\rho^{\beta}\left(\delta_{k}\right)=\chi, \quad \rho^{\beta}\left(\alpha_{i}\right)=\beta_{i} \rho\left(\alpha_{i}\right) .
$$

For the action of $u_{k}$, we have $u_{k} \cdot G\left[\alpha_{i}, v_{j}\right]=G\left[\alpha_{i}, v_{j}\right], \quad i \neq 1$. On the other hand,

$$
\begin{aligned}
u_{k} \cdot G\left[\alpha_{k}, v_{j}\right] & =G\left[\delta_{k}^{-1} \alpha_{k} \delta_{k}, v_{j}\right]=G\left[\delta_{k}^{-1}, \alpha_{k} \delta_{k}\left(v_{j}\right)\right]+G\left[\alpha_{k}, \delta_{k}\left(v_{j}\right)\right]+G\left[\delta_{k}, v_{j}\right] \\
& =G\left[\alpha_{k}, \delta_{k}\left(v_{j}\right)\right]+G\left[\delta_{k}, v_{j}\right]-G\left[\delta_{k}, \delta_{k}^{-1} \alpha_{k} \delta_{k}\left(v_{j}\right)\right]
\end{aligned}
$$

and using the formula for the action of $\delta_{k}$ which is by multiplication by $\chi$ (in particular it commutes with the $\alpha_{k}$ ),

$$
u_{k} \cdot G\left[\alpha_{k}, v_{j}\right]=\chi G\left[\alpha_{k}, v_{j}\right]+G\left[\delta_{k},\left(1-\alpha_{k}\right)\left(v_{j}\right)\right] .
$$

Finally,

$$
\begin{gathered}
u_{k} \cdot G\left[\delta_{k}, v_{j}\right]=G\left[\delta_{k}^{-1} \alpha_{k}^{-1} \delta_{k} \alpha_{k} \delta_{k}, v_{j}\right] \\
=-G\left[\delta_{k}, \delta_{k}^{-1} \alpha_{k}^{-1} \delta_{k} \alpha_{k} \delta_{k}\left(v_{j}\right)\right]-G\left[\alpha_{k}, \alpha_{k}^{-1} \delta_{k} \alpha_{k} \delta\left(v_{j}\right)\right]+G\left[\delta_{k}, \alpha_{k} \delta_{k}\left(v_{j}\right)\right] \\
+G\left[\alpha_{k}, \delta_{k}\left(v_{j}\right)\right]+G\left[\delta_{k}, v_{j}\right] \\
=-\chi G\left[\delta_{k}, v_{j}\right]-\chi^{2} G\left[\alpha_{k}, v_{j}\right]+\chi G\left[\delta_{k}, \alpha_{k}\left(v_{j}\right)\right]+\chi G\left[\alpha_{k}, v_{j}\right]+G\left[\delta_{k}, v_{j}\right] \\
=\left(\chi-\chi^{2}\right) G\left[\alpha_{k}, v_{j}\right]+G\left[\delta_{k},\left(\chi\left(\alpha_{k}-1\right)+1\right) v_{j}\right] .
\end{gathered}
$$

Lemma 4.2. Suppose $v_{j}$ is an eigenvector of $\rho\left(\alpha_{k}\right)$ with eigenvalue $r_{k, j}$. Denote $\chi:=\beta^{T}$. Then the two-dimensional subspace of $C_{1} / \partial C_{2}$ generated by $G\left[\alpha_{k}, v_{j}\right]$ and $G\left[\delta_{k}, v_{j}\right]$ is invariant under the transformation $u_{k}$, and on this subspace (with the two generators taken as basis vectors) the transformation $u_{k}$ has matrix

$$
\left.u_{k}\right|_{\left\langle G\left[\alpha_{k}, v_{j}\right], G\left[\delta_{k}, v_{j}\right]\right\rangle}=\left(\begin{array}{cc}
\chi & \left(\chi-\chi^{2}\right) \\
\left(1-\beta_{k} r_{k, j}\right) 1+\chi\left(\beta_{k} r_{k, j}-1\right)
\end{array}\right) .
$$

Proof: In the computations above, the action of $\alpha_{k}$ is by the representation $\rho^{\beta}$, and $v_{j}$ is an eigenvector of $\rho^{\beta}\left(\alpha_{k}\right)$ but this time with eigenvalue $\beta_{k} r_{k, j}$. Thus we should take the previous formulae and replace $\rho^{\beta}\left(\alpha_{k}\right) v_{j}$ by $\beta_{k} r_{k, j} v_{j}$, which gives the stated matrix.

Corollary 4.3. In the situation of the previous lemma, the eigenvalues of $u_{k}$ acting on the two dimensional subspace $\left\langle G\left[\alpha_{k}, v_{j}\right], G\left[\delta_{k}, v_{j}\right]\right\rangle$ are 1 and $\chi \beta_{k} r_{k, j}$. 
Proof: The determinant of the matrix in the lemma is

$$
\begin{aligned}
\chi+\chi^{2}\left(\beta_{k} r_{k, j}-1\right)-\left(\chi-\chi^{2}\right)\left(1-\beta_{k} r_{k, j}\right) & =\chi+\chi^{2} \beta_{k} r_{k, j}-\chi^{2}-\chi+\chi \beta_{k} r_{k, j}+\chi^{2}-\chi^{2} \beta_{k} r_{k, j} \\
& =\chi \beta_{k} r_{k, j} .
\end{aligned}
$$

The trace is $1+\chi \beta_{k} r_{k, j}$. The eigenvalues satisfy two equations which clearly hold for 1 and $\chi \beta_{k} r_{k, j}$.

Suppose $\chi \beta_{k} r_{k, j}=1$, then the matrix in the above lemma is

$$
\left(\begin{array}{cc}
\chi & \left(\chi-\chi^{2}\right) \\
\left(1-\chi^{-1}\right) & 2-\chi
\end{array}\right)=1+(\chi-1)\left(\begin{array}{cc}
1-\chi \\
\chi^{-1}-1
\end{array}\right),
$$

that is 1 plus a rank one matrix whose square is zero. In this case the $2 \times 2$ matrix of Lemma 4.2 is not semisimple. Therefore, keep the following restriction on our eigenvalues.

Convention 4.4. We assume that $\beta$ has the property that $\chi \beta_{k} r_{k, j} \neq 1$ for all eigenvalues $r_{k, j}$ of $\rho\left(\alpha_{k}\right)$. In other words, the matrix $\chi \rho^{\beta}\left(\alpha_{k}\right)$ has only nontrivial eigenvalues.

Lemma 4.5. Suppose Convention 2.1 so $\rho\left(\alpha_{k}\right)$ is a semisimple matrix, and Convention 4.4 so that $\chi \beta_{k} r_{k, j} \neq 1$. Then the monodromy transformation of $C_{1} / \partial C_{2}$ around $u_{k}$ looks up to conjugacy like

$$
\chi \rho^{\beta}\left(\alpha_{k}\right) \oplus 1^{m}
$$

where $m$ is given by a dimension count.

Proof: The map

$$
V \oplus V \rightarrow C_{1} / \partial C_{2}, \quad(u, v) \mapsto G\left[\alpha_{k}, u\right]+G\left[\delta_{k}, v\right]
$$

is injective. The action of $u_{k} \in \Upsilon$ preserves the image and there it acts as $\chi \rho^{\beta}\left(\alpha_{k}\right) \oplus 1^{r}$. Thus the multiplicity of an eigenvalue in the action of $u_{k}$ is at least as big as its multiplicity in $\chi \rho^{\beta}\left(\alpha_{k}\right)$.

On the other hand, the images of the maps $V \rightarrow H_{1}(\Gamma, V)$ given by $v \mapsto G\left[\alpha_{i}, v\right]$ for $i \neq k$, span a subspace on which $u_{k} \in \Upsilon$ acts trivially, and with the subspace of the previous paragraph, these two subspaces generate $C_{1} / \partial C_{2}$. We obtain a surjective $u_{k}$-equivariant map from a representation of the form $\chi \rho^{\beta}\left(\alpha_{k}\right) \oplus 1^{m^{\prime}}$ to $C_{1} / \partial C_{2}$. Thus, the multiplicity of a nontrivial eigenvalue in $u_{k}$ is at most 
its multiplicity in $\chi \rho^{\beta}\left(\alpha_{k}\right)$. This surjection also shows that the action of $u_{k}$ on $C_{1} / \partial C_{2}$ is semisimple.

The condition that the eigenvalues of $\chi \rho^{\beta}\left(\alpha_{k}\right)$ be all nontrivial means that the multiplicities are the same as their multiplicities in $u_{k}$. This gives the direct sum decomposition of the lemma.

Exercise 4. Calculate the action of $\zeta_{k}$. After going to the action on the homology which is the kernel of the boundary map

$$
H_{1}=Z_{1} / \partial C_{2}=\operatorname{ker}\left(C_{1} / \partial C_{2} \rightarrow C_{0} \cong V\right),
$$

the operator $\zeta_{k}$ should act by multiplication by a scalar. After tensoring with $\beta^{\cdot, b}$ it should give the identity since the local system $R C_{\beta}^{*}(L)$ doesn't depend on the choice of basepoint and hence extends across $\{b\}$.

We now consider the action of $\Upsilon$ on $H_{1}:=\operatorname{ker}\left(C_{1} / \partial C_{2} \rightarrow C_{0}\right)$.

Proposition 4.6. Suppose that $\rho$ is an irreducible representation of rank $r>2$. Suppose that the monodromy transformations $\rho\left(\alpha_{i}\right)$ are semisimple (Convention 2.1), suppose that $\chi \neq 1$ (Convention 3.1), and suppose that the eigenvalues $r_{k, j}$ of $\rho\left(\alpha_{k}\right)$ are different from $\chi^{-1} \beta_{k}^{-1}$ (Convention 4.4). The dimension of $H_{1}\left(\Gamma, \rho^{\beta}\right)$ is equal to $(n-1) r$. The group $\Upsilon$ acting on $H_{1}\left(\Gamma, \rho^{\beta}\right)$ by the raw convolution representation has the following effect on the generators:

$$
u_{k} \mapsto \beta^{V_{k}} \otimes\left(\chi \cdot \rho^{\beta}\left(\alpha_{k}\right) \oplus 1^{(n-2) r}\right) .
$$

Proof: The conditions of Proposition 2.4 apply even to the dual local system, therefore $H_{0}=0$. In particular, the boundary map is surjective onto $C_{0}$. Recall that $H_{2}=0$ because we are looking at an open curve. The Euler characteristic of the complement of $n+1$ points is $(n-1)$ which gives $\operatorname{dim} H_{1}=(n-1) r$.

On the other hand, the natural action of $\Upsilon$ on $C_{0} \cong V$ is trivial. Thus, for the monodromy transformations of the $u_{k}$, the kernel $H_{1}$ of the boundary map contains all of the nontrivial part. For this action, using the dimension count and Lemma 4.5 , the matrix of the action of $u_{k}$ is $\chi \cdot \rho^{\beta}\left(\alpha_{k}\right) \oplus 1^{(n-2) r}$. As pointed out at the start of the computation, we then have to tensor with the character $\beta^{\cdot, b}$ to get the representation corresponding to the raw convolution. 
4.4. Middling. Suppose that $\rho^{\beta}$ has some eigenvalues equal to 1 around a point $q_{i}$. The loop around that point, with the eigenvector as coefficient, gives a cycle in $H_{1}\left(\Gamma, \rho^{\beta}\right)$ which will be covariant under $\Upsilon$. Going to the middle convolution, replacing $H_{1}$ by the middle version, gets rid of these invariant cycles.

We are assuming that $\chi \neq 1$ so this behavior doesn't occur at the point $c$, and in particular the point $c$ serves as a point where there are no fixed vector so we can apply Lemma 2.6 above.

Let $F_{i} \subset L_{b}$ denote the subspace of vectors fixed by the monodromy transformation $\rho^{\beta}\left(\alpha_{i}\right)$. Since we are assuming that the local monodromy transformations are unipotent, the dimension of $F_{i}$ is equal to the multiplicity of 1 as eigenvalue of $\rho^{\beta}$. We get a map

$$
\phi_{i}: F_{i} \rightarrow H_{1}\left(\Gamma, \rho^{\beta}\right), \quad \phi_{i}(f):=G\left[\alpha_{i}, f\right] .
$$

These put together to give $\phi: \bigoplus_{i=1}^{n} F_{i} \rightarrow H_{1}\left(\Gamma, \rho^{\beta}\right)$. Recall that

$$
M H_{1}\left(\Gamma, \rho^{\beta}\right):=\frac{H_{1}\left(\Gamma, \rho^{\beta}\right)}{\phi \bigoplus_{i=1}^{k} F_{i}} .
$$

The group $\Upsilon$ acts on $M H_{1}\left(\Gamma, \rho^{\beta}\right)$. As before, we can calculate with the natural action trivialized on the basepoint section, which should then be tensored with the character $\beta^{\cdot, b}$ to obtain the middle coconvolution $M C_{\beta}^{*}(L)$.

Lemma 4.7. The map $\phi$ is equivariant for the natural action on the target, and with $u_{k}$ acting trivially on $F_{i}$ for $i \neq k$, and by multiplication by $\chi$ on $F_{k}$. Also, $\phi$ is injective.

Proof: From the previous subsection, the action of $u_{k}$ preserves $G\left[\alpha_{i}, f\right]$ for $i \neq k$. If $f$ is a fixed vector for $\rho^{\beta}\left(\alpha_{k}\right)$ then it is an eigenvector with eigenvalue $\beta_{k} r_{k, j}=1$. In the matrix of Lemma 4.2, we get that the image of $G\left[\alpha_{k}, f\right]$ is $\chi G\left[\alpha_{k}, f\right]$. Injectivity of $\phi$ follows from Lemma 2.6.

This lemma leads to the computation of the monodromy action of $u_{k}$ on the middle homology. Write $V=V^{\prime} \oplus V^{\prime \prime}$ where $\rho\left(\alpha_{k}\right)$ acts with eigenvalue $\beta_{k}^{-1}$ on $V^{\prime}$, and with eigenvalues distinct from $\beta_{k}^{-1}$ on $V^{\prime \prime}$. Thus, for $\rho^{\beta}\left(\alpha_{k}\right)$ the fixed subspace is $V^{\prime}$ with its complement $V^{\prime \prime}$. Recall 4.6 that before tensoring with $\beta^{\cdot, b}$, the natural action of $u_{k}$ on $H_{1}\left(\Gamma, \rho^{\beta}\right)$ decomposes as $\chi \rho^{\beta}\left(\alpha_{k}\right) \oplus 1^{(n-2) r}$. The underlying vector space decomposes as $V^{\prime} \oplus V^{\prime \prime} \oplus \mathbb{C}^{(n-2) r}$, and $u_{k}$ acts by $\chi$ on $V^{\prime}$, by eigenvalues different from $\chi$ on $V^{\prime \prime}$, and trivially on $\mathbb{C}^{(n-2) r}$. 
Convention 4.4 says that if $r_{k, j}$ is an eigenvalue of $\rho\left(\alpha_{k}\right)$ then $\chi \beta_{k} r_{k, j} \neq 1$. The $\beta_{k} r_{k, j}$ are the eigenvalues of $\rho^{\beta}\left(\alpha_{k}\right)$. This condition therefore says that the eigenvalues of $u_{k}$ on $V^{\prime \prime}$ are different from 1. Convention 3.1 says that $\chi \neq$ 1. Therefore the three subspaces in the above decomposition of $H_{1}\left(\Gamma, \rho^{\beta}\right)$ are distinguished by the eigenvalues of $u_{k}$.

Lemma 4.7 now implies that $\phi$ sends $F_{i}$ into the part $\mathbb{C}^{(n-2) r}$, and sends $F_{k}$ into the part $V^{\prime}$. On the other hand, $V^{\prime}$ is the space of fixed vectors of $u_{k}$, isomorphic (and indeed, equal) to $F_{k}$. Therefore, in the middle homology there is no remaining eigenspace for $\chi$, the term $V^{\prime \prime}$ remains intact, and the trivial eigenspace is reduced by an appropriate amount, to a size given by the dimension count. We can state this as follows.

Proposition 4.8. Assume Conventions 2.1, 3.1, and 4.4. The action of $u_{k} \in \Upsilon$ on the middle homology is semisimple, and we have a decomposition

$$
M H_{1}\left(\Gamma, \rho^{\beta}\right)=V^{\prime \prime} \oplus \mathbb{C}^{m},
$$

where $V^{\prime \prime}$ is the direct sum of all eigenspaces of $\rho\left(\alpha_{k}\right)$ for eigenvalues different from $\beta_{k}^{-1}$, and $m$ is given by a dimension count. The natural action of $u_{k}$ on $V^{\prime \prime}$ is by $\chi \beta_{k} \rho\left(\alpha_{k}\right)$, and the natural action on $\mathbb{C}^{m}$ is trivial. The middle convolution action is obtained by multiplying everything by $\beta^{V_{k}}$.

To put this another way, suppose $a$ is an eigenvalue of $\rho\left(\alpha_{k}\right)$ of multiplicity $m_{k}(a)$. Then the corresponding eigenvalue of the action of $u_{k}$ on the middle convolution is:

$-\beta^{V_{k}} \chi \beta^{H_{k}} a=\beta^{U_{k}} a$ with the same multiplicity $m_{k}(a)$ when $\beta^{H_{k}} a \neq 1$; or $-\beta^{V_{k}}$ with multiplicity $m_{k}(a)+\operatorname{dim} M H_{1}-r$ when $a=\left(\beta^{H_{k}}\right)^{-1}$.

This is seen by recalling that $\beta_{k}:=\beta^{H_{k}}, \chi:=\beta^{T}$ and $\beta^{U_{k}}=\beta^{V_{k}} \beta^{T} \beta^{H_{k}}$.

Exercise 5. The product of all the eigenvalues for all singular points must be 1 . As a reality-check, see that this remains true for the middle convolution with the above formulae.

4.5. The Katz transformation on the level of local monodromy. We create some notation for describing the effect of the middle convolution operation on local monodromy. Let $\mathbb{L}$ denote an abelian group with the group law written multiplicatively. Define $\operatorname{Div}(\mathbb{L})$ to be the free abelian group generated by points 
of $\mathbb{L}$. An element of $\operatorname{Div}(\mathbb{L})$ is thus a finite linear combination $g=\sum_{\alpha \in \mathbb{L}} m(\alpha) \cdot[\alpha]$ with $m(\alpha) \in \mathbb{Z}$ and $m(\alpha)=0$ for almost all $\alpha \in \mathbb{L}$. The divisor is effective if all the coefficients are positive $m(\alpha) \geq 0$.

The elements of $\mathbb{L}$ are thought of as representing possible eigenvalues, and elements of $\operatorname{Div}(\mathbb{L})$ represent conjugacy classes of semisimple matrices with these eigenvalues. The cases of interest are $\mathbb{L}=\mathbb{G}_{m}$, which applies to the Betti case of the present chapter, and $\mathbb{L}=\mathbb{A}^{1}$ which will apply for the de Rham case in the next chapter.

Since we are restricting in this paper to the case of semisimple local monodromy, we can use the simpler Div $(\mathbb{L})$ rather than the set of all Jordan normal forms [86] [91] [34] [35] [123] etc. The rank of the matrix is the degree of the divisor, that is the sum of the coefficients $m(\alpha)$. Denote this by $|g|$. Define the determinant to be $\operatorname{det}(g):=\prod_{\alpha \in \mathbb{L}} \alpha^{m(\alpha)} \in \mathbb{L}$, well defined since almost all factors are the identity element $1_{\mathbb{L}}$. For obvious reasons when the operation of $\mathbb{L}$ is conventionally denoted additively we write $\operatorname{Tr}(g)$ rather than $\operatorname{det}(g)$.

Fix $n$. A local monodromy vector is an $n$-tuple of elements of Div $(\mathbb{L})$, denoted

$$
\vec{g}=\left(g_{1}, \ldots, g_{n}\right) \in \operatorname{Div}(\mathbb{L})^{n},
$$

such that the degrees are the same, $\left|g_{1}\right|=\ldots=\left|g_{n}\right|$. Denote this common degree by $r(\vec{g})$ and call it the rank of $\vec{g}$ because it will correspond to the rank of the local system. Define the total determinant to be the product

$$
\operatorname{Det}(\vec{g}):=\operatorname{det}\left(g_{1}\right) \cdots \operatorname{det}\left(g_{n}\right) \text {. }
$$

In order to be a candidate for the local monodromy vector of a local system, we must have $\operatorname{Det}(\vec{g})=1$.

A convoluter is a function

$$
\beta: H_{1}(X-J, \mathbb{Z}) \rightarrow \mathbb{L}
$$

which, in view of the generators and relations for $H_{1}(X-J, \mathbb{Z})$, can be thought of as a vector

$$
\beta=\left(\beta^{H_{1}}, \ldots, \beta^{V_{1}}, \ldots, \beta^{U_{1}}, \ldots, \beta^{T}\right) \in \mathbb{L}^{3 n+1}
$$

subject to the relations

$$
\beta^{H_{1}} \cdots \beta^{H_{n}} \cdot \beta^{T}=1, \quad \beta^{V_{1}} \cdots \beta^{V_{n}} \cdot \beta^{T}=1, \quad \beta^{U_{i}}=\beta^{H_{i}} \beta^{V_{i}} \beta^{T}(1 \leq i \leq n) .
$$


As pointed out above in the Betti (§3.1) and de Rham (§3.2) cases, a convoluter contains the data necessary for defining a rank one object on $X$, which will also be denoted by $\beta$. The coefficients correspond to the local monodromy around the divisors $V_{i}, H_{i}, U_{i}$ and the diagonal $T$ respectively. In this picture the group $\mathbb{L}$ is the group of possible local monodromy for rank one objects, which depends on what kind of object we are considering.

The Katz operation on semisimple local monodromy assigns to a local monodromy vector $\vec{g}$ and a convoluter $\beta$ for the same number of points $n$, a new local monodromy vector $\kappa(\beta, \vec{g})$. This is defined concretely as follows.

Define the defect $\delta(\beta, \vec{g})$, which is going to be the difference between the rank of the original local system, and the rank of the new local system obtained by middle convolution. Write out the coefficients

$$
g_{i}=\sum_{\alpha} m_{i}(\alpha) \cdot[\alpha],
$$

where for clarity we denote by $[a]$ the point $a \in \mathbb{L}$ considered as a divisor. The defect is defined as

$$
\delta(\beta, \vec{g}):=(n-2) r-\sum_{i=1}^{n} m\left(\beta^{H_{i},-1}\right) .
$$

Corollary 2.5, applied to the divisor $K:=Q \cup\{c\}$ with $n+1$ points, says that

$$
\operatorname{dim} M H^{1}\left(\Gamma, \rho^{\beta}\right)=r+\delta(\beta, \vec{g}) .
$$

If no term $\beta$ is specified, it means to choose any $\beta$ such that $\beta^{H_{i},-1}:=\left(\beta^{H_{i}}\right)^{-1} \in \mathbb{L}$ is a point of maximal multiplicity for $g_{i}$, the resulting $\delta(\vec{g})$ obviously doesn't depend on which choice is made. This is the same formula as considered in $\S 2.7$.

Define the local Katz transformation at the point $q_{i}$ by

$$
\kappa_{i}(\beta, \vec{g}):=\left(m_{i}\left(\beta^{H_{i},-1}\right)+\delta(\beta, \vec{g})\right) \cdot\left[\beta^{V_{i}}\right]+\sum_{\alpha \beta^{H_{i}} \neq 1} m_{i}(\alpha) \cdot\left[\alpha \beta^{U_{i}}\right] .
$$

The global Katz transformation is defined by

$$
\vec{\kappa}(\beta, \vec{g}):=\left(\kappa_{1}(\beta, \vec{g}), \ldots, \kappa_{n}(\beta, \vec{g})\right) .
$$

Scholium 4.9. Suppose $\rho$ is a representation of rank $r$ on $Y-Q_{Y}$ satisfying Convention 2.1 that the local monodromy transformations are semisimple. Suppose $\beta$ is a convoluter, a rank one local system on $X-J$. Assume that $\beta$ satisfies 
Convention 3.1 that $\chi \neq 1$, and that Convention 4.4 holds: $\chi \rho^{\beta}\left(\alpha_{k}\right)$ have no trivial eigenvectors.

Let $\vec{g} \in \operatorname{Div}\left(\mathbb{G}_{m}\right)^{n}$ denote the vector of local monodromy data for $\rho$, and define the defect $\delta(\beta, \vec{g})$ as above.

Under these conditions, the middle coconvolution $M C_{\beta}^{*}(\rho)$ and the middle convolution $M C_{\beta}(\rho)$ are local systems on $Z-Q_{Z} \cong Y-Q_{Y}$ of rank

$$
r^{\prime}=r+\delta(\beta, \vec{g})
$$

whose local monodromy transformations are semisimple and have local monodromy types given by the Katz transformation

$$
\vec{\kappa}(\beta, \vec{g})
$$

Proof: We have done the computations for the middle coconvolution in the previous subsection. The same is true for the middle convolution by PoincaréVerdier duality. The change in ranks is formula (4.1) above, which makes the defect appear in the multiplicity of the new eigenvalue as described after Proposition 4.8 .

4.6. The Katz morphism on Betti moduli spaces. This construction extends to giving a morphism on the level of moduli spaces:

Theorem 4.10. Let $M_{B}\left(\mathbb{P}^{1}, Q ; \vec{g}\right)$ denote the Betti moduli space of local systems on $\mathbb{P}^{1}-Q$ having semisimple local monodromy transformations corresponding to $\vec{g}$. Suppose $\beta$ is a rank one local system on $(Z \times Y)-D$. Suppose that $(\beta, \vec{g})$ satisfy Conventions 3.1 and 4.4 . Then the middle convolution construction $L \mapsto M C_{\beta}(L)$ gives a morphism of moduli spaces

$$
M C_{\beta}: M_{B}\left(\mathbb{P}^{1}, Q ; \vec{g}\right) \rightarrow M_{B}\left(\mathbb{P}^{1}, Q ; \vec{\kappa}(\beta, \vec{g})\right) .
$$

This is sort of obvious, although technically speaking it requires some work: we should carry out the middle convolution construction in the context of local systems of modules over a ring. The fact that the $H^{0}$ and $H^{2}$ terms vanish, so the dimension of $H^{1}$ never jumps, is the basic thing which makes it work. Notice that our Conventions 3.1 and 4.4 are only conditions on $\beta, \vec{g}$, in particular they don't require defining open subsets of the moduli spaces. 
This type of morphism between moduli spaces was considered in [35] and other places. It is clearly related to the theory of representations of the braid group such as the Burau representation, see [98] [105].

4.7. Involutivity. One of the main properties of Katz's construction is its involutivity. This implies that it gives an isomorphism of moduli spaces. The involutivity is basic to the constitution of an algorithm: one can go forward to see if a local system with transformed local monodromy data should exist, and if one is found then one can go backward to give back a local system with the original local monodromy data.

Katz shows associativity of the convolution operator which allows him to deduce involutivity [86, 2.9.7]. Later proofs were also given in the algebraic setting by Völklein, Dettweiler-Reiter, and Crawley-Boevey and Shaw.

Katz's proof didn't rely on the Fourier transform interpretation, which nevertheless furnishes a conceptual reason for involutivity: convolution can be interpreted as a composition of two Fourier transform operators using also tensor products with rank one systems. The Fourier transform is involutive by analogy with classical real analysis, so its composition two times and also with tensoring by an invertible rank one system, is involutive with an appropriate change of convoluter as described below.

It would be interesting to use connections with irregular singularities, and "wild" harmonic theory, to make this argument precise in the complex geometric setting. This would involve Bloch-Esnault [13], Sabbah [124] and Szabo [133]. See also [15] and [67]. Very recently Aker and Szabo have contructed an involutive Nahm transform for parabolic Higgs bundles [3] which should lead to a complex analytic version of the Fourier transform construction.

For the middle convolution operation, involutivity can already be seen on the level of local monodromy data.

Proposition 4.11. Let $c: X \rightarrow X$ be the automorphism which flips the factors and let $\beta^{*}$ be the dual local system whose monodromy transformations are the inverses. Then

$$
\vec{\kappa}\left(c^{*} \beta^{*}, \vec{\kappa}(\beta, \vec{g})\right)=\vec{g}
$$


Proof: We will be making changes of variables in the sums, so it is convenient to have the following formula for the Katz transformation in terms of $g_{i}=\sum_{\alpha} m_{i}(\alpha)[\alpha]$ and the defect $d:=\delta(\beta, \vec{g})$ :

$$
\kappa_{i}(\beta, \vec{g})=\left(m_{i}\left(\beta^{H_{i},-1}\right)+d\right)\left[\beta^{V_{i}}\right]-m_{i}\left(\beta^{H_{i},-1}\right)\left[\beta^{V_{i}} \beta^{T}\right]+\sum_{\alpha} m_{i}(\alpha)\left[\alpha \beta^{U_{i}}\right] .
$$

Put $\gamma:=c^{*} \beta^{*}$. In particular we have $\gamma^{H_{i}}=\beta^{V_{i},-1}, \gamma^{V_{i}}=\beta^{H_{i},-1}, \gamma^{U_{i}}=\beta^{U_{i},-1}$, and $\gamma^{T}=\beta^{T,-1}$. Write $\overrightarrow{g^{\prime}}=\left(g_{1}^{\prime}, \ldots, g_{n}^{\prime}\right):=\vec{\kappa}(\beta, \vec{g})$ and let $r^{\prime}$ be the rank, $m^{\prime}(\alpha)$ the multiplicities and $d^{\prime}$ the defect with respect to $\gamma$. One calculates that $d^{\prime}=-d$ so the defects cancel out and at least on the level of ranks we have $\operatorname{rk}\left(\vec{\kappa}\left(\gamma, \vec{g}^{\prime}\right)\right)=r$. We can write

$$
\begin{aligned}
& \kappa_{i}\left(\gamma, \vec{g}^{\prime}\right)=\left(m_{i}^{\prime}\left(\gamma^{H_{i},-1}\right)+d^{\prime}\right)\left[\gamma^{V_{i}}\right]-\left(m_{i}^{\prime}\left(\gamma^{H_{i},-1}\right)\right)\left[\gamma^{V_{i}} \gamma^{T}\right]+\sum_{\alpha^{\prime}} m_{i}^{\prime}\left(\alpha^{\prime}\right)\left[\alpha^{\prime} \gamma^{U_{i}}\right] \\
& =m_{i}\left(\beta^{H_{i},-1}\right)\left[\beta^{H_{i},-1}\right]-\left(m_{i}\left(\beta^{H_{i},-1}\right)+d\right)\left[\beta^{V_{i}} \beta^{U_{i},-1}\right]+\sum_{\alpha} m_{i}^{\prime}\left(\alpha^{\prime}\right)\left[\alpha \beta^{U_{i},-1}\right] .
\end{aligned}
$$

The sum in the last term amounts to looking at $g_{i}^{\prime}$ but translated by $\beta^{U_{i},-1}$, in other words

$$
\begin{gathered}
\sum_{\alpha} m_{i}^{\prime}\left(\alpha^{\prime}\right)\left[\alpha \beta^{U_{i},-1}\right]= \\
\left(m_{i}\left(\beta^{H_{i},-1}\right)+d\right)\left[\beta^{V_{i}} \beta^{U_{i},-1}\right]-m_{i}\left(\beta^{H_{i},-1}\right)\left[\beta^{V_{i}} \beta^{T} \beta^{U_{i},-1}\right]+\sum_{\alpha} m_{i}(\alpha)\left[\alpha \beta^{U_{i}} \beta^{U_{i},-1}\right] .
\end{gathered}
$$

After some textual cancellation, our full expression becomes

$$
\kappa_{i}\left(\gamma, \vec{g}^{\prime}\right)=m_{i}\left(\beta^{H_{i},-1}\right)\left[\beta^{H_{i},-1}\right]-m_{i}\left(\beta^{H_{i},-1}\right)\left[\beta^{V_{i}} \beta^{T} \beta^{U_{i},-1}\right]+\sum_{\alpha} m_{i}(\alpha)[\alpha]=g_{i} .
$$

This completes the proof.

Katz has also shown by direct calculation that the virtual dimensions of the moduli spaces for $\vec{g}$ and $\vec{\kappa}(\beta, \vec{g})$ are the same.

Finally, we state the involutivity of the middle convolution morphism itself. We have seen the involutivity on the level of local monodromy data, so it makes sense to look at the composition of the middle convolution morphisms.

Theorem 4.12. The composition

$$
M_{D R}(P, Q ; \vec{g}) \stackrel{M C(\beta)}{\longrightarrow} M_{D R}(P, Q ; \kappa(\beta, \vec{g})) \stackrel{M C\left(c^{*} \beta^{*}\right)}{\longrightarrow} M_{D R}(P, Q ; \vec{g})
$$

is the identity, if we are in the situation of Theorem 4.10 for both of the morphisms. 
We don't describe the proof here but refer to Katz [86], Völklein [139], DettweilerReiter [44], and more recently Crawley-Boevey and Shaw [35].

4.8. Detecting emptiness of the moduli space. One of the main features of Katz's construction is that it permits us to detect whether a given moduli space is empty or not in terms of the next moduli space in the algorithm. In other words,

$$
M_{B}(\vec{g})=\emptyset \Leftrightarrow M_{B}(\kappa(\beta, \vec{g}))=\emptyset
$$

assuming Conventions 3.1 and 4.4.

This is specially the case when $M_{B}(\kappa(\beta, \vec{g}))$ is not even defined because one of the divisors in the vector $\kappa(\beta, \vec{g})$ is no longer effective. It is comforting to work this case out explicitly. Let $d=\delta(\beta, \vec{g})$ denote the defect. The multiplicities in the local divisors $\kappa_{i}\left(\beta, d, g_{i}\right)$ are either the same as in $g_{i}$, or else they are changed by adding $d$. In particular, if $d \geq 0$ then we will never get to a noneffective divisor. Thus we may assume that $d<0$. Suppose that $\kappa_{i}\left(\beta, d, g_{i}\right)$ becomes noneffective. The only multiplicity which changes is $m_{i}\left(\beta^{H_{i},-1}\right)$, which becomes

$$
m_{i}\left(\beta^{H_{i},-1}\right)+d \text {. }
$$

In particular, we are in the current situation, only if

$$
m_{i}\left(\beta^{H_{i},-1}\right)+d<0 .
$$

Plugging in the formula for the defect, we have

$$
m_{i}\left(\beta_{H_{i}}^{-1}\right)+r(n-2)-\sum_{j=1}^{n} m_{j}\left(\beta^{H_{i},-1}\right)<0,
$$

and adding $r$ to both sides and simplifying we get

$$
\sum_{j \neq i}\left(r-m_{j}\left(\beta^{H_{i},-1}\right)\right)<r .
$$

This says that the sum for $j \neq i$ of the ranks of the matrices $\rho^{\beta}\left(\alpha_{j}\right)-1$ is $<r$. Since these matrices generate the action of the group algebra on the vector space $V$, under this condition the action cannot be irreducible. So, $\rho^{\beta}$ and hence $\rho$ is not irreducible. Thus, we have the following lemma.

Lemma 4.13. Suppose that $\vec{g}$ consists of effective divisors, and at least one of the divisors in $\kappa(\beta, \vec{g})$ is not effective. In this case, the representation $\rho$ cannot 
be irreducible. In the case where $\vec{g}$ is automatically irreducible, this means that the moduli space $M_{B}(\vec{g})$ is empty.

4.9. Running Katz's algorithm (Kostov's program). Kostov invented the protocol of applying Katz's algorithm to the nonrigid case. Suppose $\vec{g}$ is a local monodromy vector. Choose a convoluter $\beta$ so that $\left(\beta^{H_{i}}\right)^{-1}$ is an eigenvalue of maximal multiplicity for $g_{i}$. Thus $\delta(\beta, \vec{g})=\delta(\vec{g})$. If $\delta(\vec{g})<0$ and if the pair $(\beta, \vec{g})$ satisfies Conventions 3.1 and 4.4 , then we obtain an isomorphism of moduli spaces for $\vec{g}$ and the Katz-transformed vector $\vec{\kappa}(\beta, \vec{g})$. The rank strictly decreases, so we can keep going on in the same way, until we get to $r=1$ or more generally to a case where all of the local monodromy matrices are diagonal; to an impossibility result; to the problem discussed in the subsequent paragraph below; or until we get into the range $\delta \geq 0$. If we hit an impossibility result anywhere along the way, then the original moduli space was empty. If we hit $r=1$ then the original moduli space was a point. If we get into the range $\delta \geq 0$ then according to Kostov we expect that the moduli space should be nonempty, with a direct construction of some points [91] [92]. Crawley-Boevey and Shaw [35] gave a different construction covering cases not treated in [91] [92], and prove in some cases that the moduli space is a complete intersection. We will discuss a Higgs-bundle version of the direct construction in $\S 6.3$ below.

The problem with the previous paragraph is that somewhere along the way, we might hit a vector $\vec{g}$ for which every choice of $\beta$ corresponding to maximal multiplicities, dissatisfies either Convention 3.1 or Convention 4.4. In this case the algorithm no longer makes sense as we have described it. Apparently it can be made to work anyway, but this goes beyond the scope of the present discussion and we refer to the papers of Kostov and Crawley-Boevey. Instead, we will just point out that it doesn't happen if the original eigenvalues are sufficiently general.

In Kostov's notation, a "nongenericity relation" is a subset of the eigenvalues counted with multiplicities, of the same rank $r^{\prime} \in 1, \ldots, r-1$ at each point $q_{i}$, such that the product of them all is 1 . Any nontrivial sub-local system has monodromy sub-data which give a nongenericity relation. 
Kostov says that a monodromy data vector $\vec{g}$ is 1-generic if there is no nongenericity relation of rank $r^{\prime}=1$. This is the same as saying that there is no equation $a_{1} \cdots a_{n}=1$ such that $a_{i}$ is an eigenvalue of $g_{i}$.

Lemma 4.14. Suppose $\vec{g}$ is 1-generic, and suppose $\beta$ is a convoluter such that each $\left(\beta^{H_{i}}\right)^{-1}$ is an eigenvalue of $g_{i}$. Then the pair $(\beta, \vec{g})$ satisfies Conventions 3.1 and 4.4 and we get a middle convolution isomorphism between moduli spaces.

Proof: It is trivial that the pair satisfies the conditions. In order to get an isomorphism we also need to have the same conditions for the inverse pair $\left(c^{*} \beta^{*}, \vec{\kappa}(\beta, \vec{g})\right)$. Convention 3.1 for $c^{*} \beta^{*}$ is equivalent to Convention 3.1 for $\beta$. For Convention 4.4 note that the eigenvalues of $\kappa_{i}(\beta, \vec{g})$ are either $\varphi=\beta^{U_{i}} \alpha$ for eigenvalues $\alpha$ of $g_{i}$ with $\beta^{H_{i}} \alpha \neq 1$, or else $\varphi=\beta^{V_{i}}$. Convention 4.4 for the inverse pair thus requires for these $\varphi$

$$
\left(c^{*} \beta^{*}\right)^{T}\left(c^{*} \beta^{*}\right)^{H_{i}} \varphi \neq 1 .
$$

Recalling that $\left(c^{*} \beta^{*}\right)^{T}\left(c^{*} \beta^{*}\right)^{H_{i}}=\beta^{T,-1} \beta^{V_{i},-1}$, the condition becomes

$$
\begin{gathered}
\beta^{T,-1} \beta^{V_{i},-1} \cdot \beta^{U_{i}} \alpha \neq 1, \quad \text { for } \beta^{H_{i}} \alpha \neq 1, \\
\beta^{T,-1} \beta^{V_{i},-1} \cdot \beta^{V_{i}} \neq 1 .
\end{gathered}
$$

The first is verified by tautology and the second is Convention 3.1.

In Katz's original rigid case, a nongenericity relation among eigenvalues of highest multiplicity automatically causes the local system to become reducible, and meeting such a nongenericity relation anywhere along the way rules out existence of any irreducible rigid local system. I would like to thank the referee for pointing out the following very interesting example, which shows that there can be a nongenericity relation among other eigenvalues, even for an irreducible rigid local system. The example consists of a local system of rank 3 with 3 singular points having local monodromy eigenvalues $(a, b, c),(u, v, w),(g, h, h)$. It is rigid, and exists even with a nongenericity relation of the form $a u g=1$. If there is no other nongenericity relation then the local system cannot be reducible (by looking at the block of size 2). One can construct this system by convolution of a hypergeometric system $\left(a^{\prime}, b^{\prime}\right),\left(u^{\prime}, v^{\prime}\right),\left(g^{\prime}, h^{\prime}\right)$ with a convoluter having $\beta^{H_{i}}=$ $\beta^{V_{i}}=x, y$, or $z=\left(h^{\prime}\right)^{-1}(i=1,2$, or 3$)$. As an exercise in applying the Katz 
transformation, the convoluted system is

$$
\left(\begin{array}{c}
a^{\prime} x y^{-1} z^{-1} \\
b^{\prime} x y^{-1} z^{-1} \\
x
\end{array}\right),\left(\begin{array}{c}
u^{\prime} y x^{-1} z^{-1} \\
v^{\prime} y x^{-1} z^{-1} \\
y
\end{array}\right),\left(\begin{array}{c}
g^{\prime} z x^{-1} y^{-1} \\
z \\
z
\end{array}\right) .
$$

Thus aug $=a^{\prime} x y^{-1} z^{-1} u^{\prime} y x^{-1} z^{-1} g^{\prime} z x^{-1} y^{-1}=a^{\prime} u^{\prime} g^{\prime} x^{-1} y^{-1} z^{-1}$ can be equal to 1 by an appropriate choice of $x, y, z$.

For nonrigid local systems the situation is even less clear and we will be happy with the following result.

Proposition 4.15. Fix Kostov's polymultiplicity vector (PMV) [89] etc. containing the multiplicities of eigenvalues in the divisors $g_{i}$. The variety of all possible $\vec{g}$ with this polymultiplicity vector, is a disjoint union of d connected open subsets of tori, where $d$ is the pgcd of all the multiplicities in $\vec{g}$. If $\vec{g}$ is a sufficiently general point in any of these connected components, then we can run Katz's algorithm until we hit either an empty moduli space for the reason discussed in $\S 4.8$, or the case of all diagonal local monodromy (i.e. rank one system tensored with $\left.\mathbb{C}^{r}\right)$, or the case $\delta \geq 0$ which will be discussed in $\S 6.3$ below. The monodromy vectors encountered along the way are always themselves general points, in particular they are 1-generic.

Proof: Invertibility of the transformation on local monodromy data (Lemma 4.11) plus its continuity with respect to the eigenvalue parameters if the PMV is fixed, imply that for $\vec{g}$ general in its connected component, and $\beta$ general in the variety of possible choices given that the $\beta^{H_{i}}$ come from $\vec{g}$ (that is, general among the possible choices of $\left.\beta^{V_{i}}\right)$, the resulting $\vec{\kappa}(\beta, \vec{g})$ is again general in its connected component. Thus, formally applying a sequence of Katz transformations as for the algorithm, we encounter only general local monodromy vectors.

If the PMV is not simple, that is if the pgcd of all the multiplicities is $d \geq 2$, then there can exist a nongenericity relation even for general $\vec{g}$. However, the nongenericity relation is always of rank at least $r / d$, and the case $d=r$ is the degenerate one with only diagonal matrices. Thus, a general $\vec{g}$ in any connected component is always 1-generic, except in the degenerate diagonal case.

In case of a non-simple PMV, the variety in the previous proposition has some components where there is a nongenericity relation. If the moduli space has dimension 2, when we get to $\delta=0$ and $\sigma=0$ Kostov shows in [90] that all local 
systems are reducible for the nongeneric components. The case of dimension 2 is somewhat special and is not covered by our construction in $\S 6.3$.

Roberts studies the geographical implications of Katz's algorithm in the rigid case [123], and it would be good to extend his results to the nonrigid case.

\section{Middle COnvolution-The De Rham Version}

The de Rham version involves replacing local systems by logarithmic connections [116] [13] [75]. Middle convolution in the "Fuchsian" case of connections on the trivial bundle has been extensively considered [89] etc., [63] [64] [45] [46] [55] [33]. In our treatment we don't distinguish between trivial and nontrivial underlying bundles, so in a certain sense we consider less information than these references, on the other hand our approach places things in an abstract setting.

In order to use the logarithmic de Rham complex, it is essential to have a morphism between smooth projective varieties with normal crossings divisors. Thus we use the blowing-up $X$ with its divisor $J \subset X$ described in $\S 3$. The second projection gives a map $\xi:(X, J) \rightarrow(Z, Q)$ in good position, meaning that the inverse image of $Q$ is the divisor $U+V \subset J$ which has normal crossings.

For a vector bundle with logarithmic connection $(E, \nabla)$ on $\left(Y, Q_{Y}\right)$ and a de Rham convoluter $\beta \in H^{0}\left(X, \Omega_{X}^{1}(\log J)\right)$, define a vector bundle with logarithmic connection on $X$ :

$$
\left(F, \nabla_{F}\right):=\eta^{*}(E, \nabla) \otimes\left(\mathcal{O}_{X}, d+\beta\right) .
$$

The divisor $H T:=H_{1}+\ldots+H_{n}+T \subset J$ is transverse to the fibers of $\xi$. In a relative version of the discussion of $\S, 2.3$ we can define the middle relative de Rham complex with respect to $H T$, by the exact sequence

$$
0 \rightarrow M D R(X / Z, F ; H T) \rightarrow D R(X / Y, F) \rightarrow \mathcal{F}_{H T / Z}^{0}[-1] \rightarrow 0 .
$$

For $z \in Z$, denote by $X_{z}$ the fiber of $\xi$ over $z$, and let

$$
\operatorname{MDR}\left(X_{z}, F ; H T_{z}\right):=\left.\operatorname{MDR}(X / Z, F ; H T)\right|_{X_{z}}
$$

with similar notation for the full de Rham complex. Over points $z \in Z-Q$ this is the same thing as the middle de Rham complex for $\left(X_{z}, H T_{z}\right) \cong(Y, Q+\{z\})$ considered in $\S 2.3$. In order to have a good base-change theory, we impose the following. 
Convention 5.1. For every $z \in Z$, the degree 0 and 2 hypercohomology groups of the restriction $M D R\left(X_{z}, F ; H T_{z}\right)$ vanish.

This condition implies that $\mathbb{R}^{1} \xi_{*} M D R(X / Z, F ; H T)$ is locally free over $Z$ with fiber over a point $z$ equal to $\mathbb{H}^{1} M D R\left(X_{z}, F ; H T_{z}\right)$. It has a logarithmic GaussManin connection denoted by $\nabla_{G M \text {,mid }}$, and we define the de Rham middle convolution as

$$
M C_{\beta}(E, \nabla):=\left(\mathbb{R}^{1} \xi_{*} M D R(X / Z, F ; H T), \nabla_{G M, \text { mid }}\right),
$$

a vector bundle with logarithmic connection on $\left(Z, Q_{Z}\right)$.

The restriction of the quotient term in (5.1) to a point $z \in Z$ is just a skyscraper sheaf placed in cohomological degree 1 , so it has no $\mathbb{H}^{0}$ or $\mathbb{H}^{2}$. The long exact sequence for the higher derived direct image of the exact sequence (5.1) therefore gives the following.

Lemma 5.2. Suppose that Convention 5.1 holds. Then the same vanishing holds for the full de Rham complex, the $\mathbb{R}^{1} \xi_{*} D R(X / Z, F)$ is again a vector bundle compatible with base change, and we have a short exact sequence

$$
0 \rightarrow \mathbb{R}^{1} f_{*} M D R(X / Z, F ; H T) \rightarrow \mathbb{R}^{1} f_{*} D R(X / Z, F) \rightarrow \mathbb{R}^{0} f_{*}\left(\mathcal{F}_{H T / Z}^{0}\right) \rightarrow 0 .
$$

This short exact sequence is compatible with the Gauss-Manin connections $\nabla_{G M \text {,mid }}$ on the left and $\nabla_{G M}$ in the middle.

The classical definition of the Gauss-Manin connection is as the connecting map for the short exact sequence of complexes

$$
0 \rightarrow D R(X / Z, F) \otimes \xi^{*} \Omega_{Z}^{1}(\log Q)[-1] \rightarrow D R(X, F) \rightarrow D R(X / Z, F) \rightarrow 0 .
$$

When $q \in Q$ is a singular point, the de Rham complex $D R(X, F)$, which by convention means the logarithmic de Rham complex with respect to $J$, can be restricted to a complex $\left.D R(X, F)\right|_{X_{q}}$ on the fiber $X_{q} \subset J$. We obtain a restriction of (5.3) to $X_{q}$. Note that $\Omega_{Z}^{1}(\log Q)_{q} \cong \mathbb{C}$ and the residue of $\nabla_{G M}$ at $q$ is the endomorphism

$$
\mathbb{H}^{1} D R\left(X_{q}, F\right) \rightarrow \mathbb{H}^{2}\left(D R\left(X_{q}, F\right) \otimes_{\mathbb{C}} \Omega_{Z}^{1}(\log Q)_{q}[-1]\right)=\mathbb{H}^{1} D R\left(X_{q}, F\right)
$$

induced by the connecting map for the restriction of (5.3). 
The expression as a connecting map is not very convenient for calculating the eigenvalues. The calculation was done by Katz in [84] (thanks to H. Esnault for pointing out this reference). Without going through all of the details, here is the conclusion. In our case, $q=q_{i}$ for some $i=1, \ldots, n$, and the singular fiber $X_{q}$ consists of two components $X_{q}=U_{i} \cup V_{i}$ meeting in a point $w_{i}:=U_{i} \cap V_{i}$. We have a short exact sequence

$$
0 \rightarrow D R\left(U_{i}, F_{U_{i}}\left(-w_{i}\right)\right) \rightarrow D R\left(X_{q}, F_{X_{q}}\right) \rightarrow D R\left(V_{i}, F_{V_{i}}\right) \rightarrow 0 .
$$

Note that $H T$ meets $X_{q}$ in a collection of smooth points distinct from the crossing point $w_{i}$. Thus the exact sequence defining the middle de Rham complex is compatible with (5.4), and we have the same short exact sequence for middle de Rham complexes

$0 \rightarrow M D R\left(U_{i}, F_{U_{i}}\left(-w_{i}\right), H T_{U_{i}}\right) \rightarrow M D R\left(X_{q}, F_{X_{q}}, H T_{X_{q}}\right) \rightarrow M D R\left(V_{i}, F_{V_{i}}, H T_{V_{i}}\right) \rightarrow 0$.

We refine Convention 5.1 to apply to each of the components:

Convention 5.3. For $j=0,2$ we require that

$$
\mathbb{H}^{j} M D R\left(U_{i}, F_{U_{i}}\left(-w_{i}\right), H T_{U_{i}}\right)=0, \quad \mathbb{H}^{j} M D R\left(V_{i}, F_{V_{i}}, H T_{V_{i}}\right)=0 .
$$

Assuming Convention 3.2, this condition for all the $q_{i}$ implies Convention 5.1. For points $z \in Z-Q$, Lemma 2.8 provides the required vanishing.

Proposition 5.4. Assuming Convention 5.3, we get a short exact sequence from (5.5) on the level of $\mathbb{H}^{1}$. The residues of $\nabla_{F}$ along $U_{i}$ and $V_{i}$ give endomorphisms of $F_{U_{i}}$ and $F_{V_{i}}$. These fit into a diagram

$$
\begin{aligned}
& 0 \rightarrow \mathbb{H}^{1} M D R\left(U_{i}, F_{U_{i}}\left(-w_{i}\right)\right) \rightarrow \mathbb{H}^{1} M D R\left(X_{q}, F_{X_{q}}\right) \rightarrow \mathbb{H}^{1} M D R\left(V_{i}, F_{V_{i}}\right) \rightarrow 0 \\
& \downarrow \\
& 0 \rightarrow \mathbb{H}^{1} M D R\left(U_{i}, F_{U_{i}}\left(-w_{i}\right)\right) \rightarrow \mathbb{H}^{1} M D R\left(X_{q}, F_{X_{q}}\right) \rightarrow \mathbb{H}^{1} M D R\left(V_{i}, F_{V_{i}}\right) \rightarrow 0
\end{aligned}
$$

where the endomorphism of $\mathbb{H}^{1} D R\left(X_{q}, F_{X_{q}}\right)$ is the residue of the middle GaussManin connection $\nabla_{G M \text {,mid }}$ at $q=q_{i}$. For brevity the notations $H T_{U_{i}}$ etc. have been omitted.

In our case, the endomorphisms of the left and right terms will be semisimple. This will imply that the residue of $\nabla_{G M \text {,mid }}$ is semisimple, as long as we know that the endomorphisms on the left and right don't have any common eigenvalues. We can state this as the following lemma. 
Lemma 5.5. Let $\psi_{U_{i}}$ and $\psi_{V_{i}}$ be the endomorphisms of $\mathbb{H}^{1} M D R\left(U_{i}, F_{U_{i}}\left(-w_{i}\right), H T_{U_{i}}\right)$ and $\mathbb{H}^{1} M D R\left(V_{i}, F_{V_{i}}, H T_{V_{i}}\right)$ respectively, determined by the endomorphisms of $F_{U_{i}}$ and $F_{V_{i}}$ given by the residues of $\nabla$ along $U_{i}$ and $V_{i}$. Suppose that these endomorphisms are semisimple, and don't have any common eigenvalues. Then the residue of $\nabla_{G M \text {,mid }}$ at $q_{i}$ is semisimple and isomorphic to $\psi_{U_{i}} \oplus \psi_{V_{i}}$.

On $U_{i}$ and $V_{i}$ we have a logarithmic structure also at the point $w_{i}$. However, this point is not included in the "middle" part which is just $H T_{U_{i}}$ or $H T_{V_{i}}$.

We now turn to the fact that $F$ is the pullback of $(E, \nabla)$, tensored with $\left(\mathcal{O}_{X}, d+\right.$ $\beta)$. From the above discussion, the main problem is to calculate the restrictions

$$
\left.\eta^{*}(E, \nabla)\right|_{U_{i}},\left.\quad \eta^{*}(E, \nabla)\right|_{V_{i}},\left.\quad \beta\right|_{U_{i}},\left.\quad \beta\right|_{V_{i}} .
$$

We can define the restriction of a logarithmic one-form $\beta$ to $V_{i}$ as follows. It depends on the pullback of the coordinate function $t$ from $Z$ (where $t\left(q_{i}\right)=0$ ). Set $b:=\operatorname{res}\left(\beta, V_{i}\right)$, then $\beta-b \frac{d t}{t}$ is a logarithmic form having zero residue along $V_{i}$, thus it is in the kernel of the residue map on logarithmic forms which maps by restriction to $\Omega_{V_{i}}^{1}(\log )$. Define

$$
\left.\beta\right|_{V_{i}} ^{t}:=\left.\left(\beta-b \frac{d t}{t}\right)\right|_{V_{i}} .
$$

It is a logarithmic form on $V_{i}$ whose residues along $H_{j} \cap V_{i}$ are just $\beta^{H_{j}}$, for $j \neq i$. This determines the restriction, and it has residue at the intersection point

$$
\operatorname{res}\left(\left.\beta\right|_{V_{i}} ^{t}, w_{i}\right)=-\sum_{j \neq i} \beta^{H_{j}}
$$

The similarly-defined restriction $\left.\beta\right|_{U_{i}} ^{t}$ is a logarithmic form on $U_{i}$ whose residues along $H_{i} \cap U_{i}$ and $T \cap U_{i}$ are respectively $\beta^{H_{i}}$ and $\beta^{T}$, so

$$
\operatorname{res}\left(\left.\beta\right|_{U_{i}} ^{t}, w_{i}\right)=-\beta^{H_{i}}-\beta^{T} .
$$

The relation $\beta^{T}+\sum_{i} \beta^{H_{i}}=0$ gives $\operatorname{res}\left(\left.\beta\right|_{V_{i}} ^{t}, U_{i} \cap V_{i}\right)+\operatorname{res}\left(\left.\beta\right|_{U_{i}} ^{t}, U_{i} \cap V_{i}\right)=0$, characteristic of the fact that these restrictions correspond to a single logarithmic form on $X_{q_{i}}=U_{i} \cup V_{i}$.

Now restrict $\left(F, \nabla_{F}\right)$ to $V_{i}$. Since $\left.\eta\right|_{V_{i}}$ is the identity, $\left.\eta^{*}(E, \nabla)\right|_{V_{i}} \cong(E, \nabla)$. The restriction of $F$ is therefore

$$
\left.\left(F, \nabla_{F}\right)\right|_{V_{i}}=\left.\left(\eta^{*} E, \eta^{*} \nabla+\beta\right)\right|_{V_{i}}=\left(E, \nabla+\left.\beta\right|_{V_{i}} ^{t}\right) .
$$


The residual endomorphism induced by $\nabla_{F}$ is just scalar multiplication by $\beta^{V_{i}}$.

Next look at the restriction of $\left(F, \nabla_{F}\right)$ to $U_{i}$. It clearly depends only on the local form of $(E, \nabla)$ near the point $q_{i}$. We may even localize in an analytic neighborhood, and so assume that $E$ has the form of a trivial bundle $\mathcal{O}^{r}$ and the connection is given by $\nabla=d+A \frac{d y}{y}$. We use the notation $y$ for our coordinate on $Y$ at the point $q_{i}$ (which should be the same as $t$ under $Y \cong Z$ ), also considered as a function on $Z \times Y$ or $X$.

Now $t$ and $y$ give coordinates on $Z \times Y$. The ratio $u=y / t$ is a coordinate on $X$, in a neighborhood of the point $H_{i} \cap U_{i}$ along $U_{i}$. On $U_{i}$ it corresponds to the linear coordinate which takes the values $u\left(U_{i} \cap H_{i}\right)=0, u\left(U_{i} \cap T\right)=1$, $u\left(U_{i} \cap V_{i}\right)=\infty$.

The relation $\frac{d y}{y}=\frac{d u}{u}+\frac{d t}{t}$ allows us to calculate the restriction

$$
\left.\left(\frac{d y}{y}\right)\right|_{U_{i}} ^{t}=\frac{d u}{u} .
$$

The residue of the pullback of $\frac{d y}{y}$ along $U_{i}$ is equal to 1 . Using $(E, \nabla) \cong\left(\mathcal{O}^{r}, d+\right.$ $\left.A \frac{d y}{y}\right)$ we get that the restriction of the pullback to $U_{i}$ is

$$
\left.\left(\eta^{*} E, \eta^{*} \nabla+\beta\right)\right|_{U_{i}}=\left(\mathcal{O}_{U_{i}}^{r}, d+A \frac{d u}{u}+\left.\beta\right|_{U_{i}} ^{t}\right),
$$

and the residue of $\eta^{*} \nabla+\beta$ along $U_{i}$ is $A+\beta^{U_{i}}$ which is an endomorphism of this bundle preserving the logarithmic connection. Here, in canonical terms $\mathcal{O}^{r}$ corresponds to the fiber $E_{q_{i}}$ and $A$ corresponds to the residue of $\nabla$ at $q_{i}$.

Apply Lemma 5.5 to calculate the residue of $\nabla_{G M \text {,mid }}$. By Convention 2.2, $A$ is semisimple with eigenvalues never differing by a nonzero integer. Invoking either Corollary 2.8 using the fact that the residue of $\left.\beta\right|_{U_{i}} ^{t}$ at $T_{U_{i}}$ is a nonzero scalar (Convention 3.2), or just by direct computation, we have

$$
\mathbb{H}^{i} D R\left(\mathcal{O}_{U_{i}}\left(-U_{i} \cap V_{i}\right)^{r}, d+A \frac{d u}{u}+\left.\beta\right|_{U_{i}} ^{t}\right)=0, \quad i=0,2 .
$$

A direct computation using the fact that $\Omega_{U_{i}}^{1}(\log )\left(-w_{i}\right) \cong \mathcal{O}_{U_{i}}$ gives

$$
\mathbb{H}^{1} D R\left(\mathcal{O}_{U_{i}}\left(-U_{i} \cap V_{i}\right)^{r}, d+A \frac{d u}{u}+\left.\beta\right|_{U_{i}} ^{t}\right) \cong H^{0}\left(\Omega_{U_{i}}^{1}(\log )\left(-w_{i}\right) \cong \mathcal{O}_{U_{i}}\right)^{r}=\mathbb{C}^{r},
$$

and the action of the residue of $\eta^{*} \nabla+\beta$ is given by the matrix $A+\beta^{U_{i}}$.

The residue is nontrivial on the diagonal (Convention 3.2) so the middle condition at $U_{i} \cap T$ has no effect, and the middle condition at the point $U_{i} \cap H_{i}$ removes 
the zero eigenspace of the residue there, that is to say the zero eigenspace of the matrix $A+\beta^{H_{i}}$. Introduce the following notation: if $M$ is a semisimple matrix then $M^{\dagger}$ is the same endomorphism but only of the sum of eigenspaces different from zero. Thus the contribution from $U$ to the residue of $\nabla_{G M \text {,mid }}$ on the middle direct image is

$$
\operatorname{res}\left(\nabla_{G M, \text { mid }}\right)_{U}=\left(A+\beta^{H_{i}}\right)^{\dagger}+\left(\beta^{U_{i}}-\beta^{H_{i}}\right) .
$$

Recall that $\beta^{U_{i}}=\beta^{H_{i}}+\beta^{T}+\beta^{V_{i}}$, giving

$$
\operatorname{res}\left(\nabla_{G M, \text { mid }}\right)_{U}=\left(A+\beta^{H_{i}}\right)^{\dagger}+\beta^{V_{i}}+\beta^{T} .
$$

The contribution from $V$ is the cohomology of $\nabla+\left.\beta\right|_{V_{i}} ^{t}$, with middle condition at the points $H_{j} \cap V_{i}$ for $j \neq i$ and no middle condition at $w_{i}=U_{i} \cap V_{i}$. This contribution occurs with a single eigenvalue which is the residue of $\beta$, in our notation $\beta^{V_{i}}$. Let $d_{i}$ denote the dimension of this cohomology group and $\mathbf{1}^{d_{i}}$ is the identity matrix of rank $d_{i}$. If Lemma 5.5 can be applied then we conclude that the full residue of the Gauss-Manin connection on the middle convolution is given by

$$
\operatorname{res}\left(\nabla_{G M, \text { mid }}\right)=\left[\left(A+\beta^{H_{i}}\right)^{\dagger}+\beta^{V_{i}}+\beta^{T}\right] \oplus\left[\beta^{V_{i}} \mathbf{1}^{d_{i}}\right] .
$$

In order to be able to apply Lemma 5.5 we need to know that the eigenvalues of the two pieces don't coincide. We also need something for the first sentence in Corollary 2.8. The following condition is analogous to Convention 4.4 from the Betti case.

Convention 5.6. For any eigenvalue $\alpha$ of the residue $A=\operatorname{res}\left(\nabla, q_{i}\right)$, we have

$$
\alpha+\beta^{H_{i}}+\beta^{T} \notin \mathbb{Z}, \quad \alpha+\beta^{H_{i}} \notin \mathbb{Z}-\{0\} .
$$

The first condition is equivalent to saying $\alpha-\sum_{j \neq i} \beta^{H_{j}} \notin \mathbb{Z}$, and if each $-\beta^{H_{j}}$ is an eigenvalue of the residue at $q_{j}$ then this condition would be a consequence of 1-genericity as in 4.14. The second condition will hold whenever we need to choose $-\beta^{H_{i}}$ from among the eigenvalues of $\operatorname{res}\left(\nabla, q_{i}\right)$, by Convention 2.2.

Lemma 5.7. Suppose Conventions 2.2, 3.2 and 5.6 hold. Then the eigenvalues of $\left(A+\beta^{H_{i}}\right)^{\dagger}+\beta^{V_{i}}+\beta^{T}$ are distinct from $\beta^{V_{i}}$, so Lemma 5.5 can be applied as above (5.6) with

$$
d_{i}=(n-2) r-\sum_{j \neq i} m_{j}\left(-\beta^{H_{j}}\right)
$$


Proof: The eigenvalues of $\left(A+\beta^{H_{i}}\right)^{\dagger}+\beta^{V_{i}}+\beta^{T}$ are of the form $\alpha+\beta^{H_{i}}+\beta^{T}+\beta^{V_{i}}$ for $\alpha$ eigenvalues of $A$. Under Convention 5.6 these are different from $\beta^{V_{i}}$. For the dimension of the piece coming from $V_{i}$, note that

$$
\operatorname{res}\left(\nabla+\left.\beta\right|_{V_{i}} ^{t}, w_{i}\right)=A+\operatorname{res}\left(\left.\beta\right|_{V_{i}} ^{t}, w_{i}\right)=A-\sum_{j \neq i} \beta^{H_{j}}=A+\beta^{H_{i}}+\beta^{T} .
$$

Convention 5.6 says that the eigenvalues here are never integers, also the residues at points of $H_{V_{i}}$ are never nonzero integers. Thus Corollary 2.8 applies and we can calculate the dimension $d_{i}$ by using the Euler characteristic which gives the formula as stated. The terms in the sum over $i \neq j$ come from the middle conditions at the points $H_{j} \cap V_{i}$; there is no middle condition at the remaining point $w_{i}$.

As in $\S 2.5$ and $\S 4.5$ above, let $\vec{g}=\left(g_{1}, \ldots, g_{n}\right)$ denote the residual data for $(E, \nabla)$ with $g_{i} \in \operatorname{Div}\left(\mathbb{A}^{1}\right)$ effective divisors representing the multiplicity vectors of the eigenvalues. Asking that the residues lie in conjugacy classes $\mathbf{c}\left(g_{i}\right)$ insures Convention 2.2 automatically, and Conventions 3.2 and 5.6 are conditions only on the pair $(\beta, \vec{g})$. The result of Lemma 5.5 applied as in (5.6) says exactly that the vector of residual data for $\nabla_{G M \text {,mid }}$ is given by $\vec{\kappa}(\beta, \vec{g})$. We can sum up as follows.

Scholium 5.8. Suppose $(E, \nabla)$ is a logarithmic connection on $\left(Y, Q_{Y}\right)$ with semisimple residues (Convention 2.2) corresponding to a vector $\vec{g} \in \operatorname{Div}\left(\mathbb{A}^{1}\right)^{n}$, and suppose $\beta$ is a de Rham convoluter (§3.2). Suppose that Convention 3.2) that $\beta^{T} \notin \mathbb{Z}$, and Convention 5.6 that $\alpha+\beta^{H_{i}}+\beta^{T} \notin \mathbb{Z}$ and $\alpha+\beta^{H_{i}} \notin \mathbb{Z}-\{0\}$ for any eigenvalue $\alpha$ of $\operatorname{res}\left(\nabla, q_{i}\right)$. These are conditions on $(\beta, \vec{g})$ only. Then the de Rham middle convolution $M C_{\beta}(E, \nabla)$ is a logarithmic connection on $\left(Z, Q_{Z}\right)$ with semisimple residues whose vector of residual data is given by the Katz transformation $\vec{\kappa}(\beta, \vec{g})$.

Theorem 5.9. Suppose $Q \subset \mathbb{P}^{1}$ is a set of $n$ points, $\vec{g} \in \operatorname{Div}\left(\mathbb{A}^{1}\right)^{n}$ is a vector of semisimple residual data, and $\beta$ is a de Rham convoluter. Suppose Conventions 3.2 and 5.6 hold. Then middle convolution induces an isomorphism

$$
M C_{\beta}: M_{D R}\left(\mathbb{P}^{1}, Q ; \vec{g}\right) \stackrel{\cong}{\rightrightarrows} M_{D R}\left(\mathbb{P}^{1}, Q ; \vec{\kappa}(\beta, \vec{g}) .\right.
$$

This isomorphism is involutive like in $\S 4.7$ (but with $-c^{*} \beta$ instead of $c^{*} \beta^{*}$ ) and is compatible with the isomorphism of Theorem 4.10 via the Riemann-Hilbert correspondence [45, Theorem 1.2]. 


\section{HARmonic Bundles AND PARABolic STRUCTURES}

There is a notion of parabolic bundle on $Z$ with parabolic structures at the $q_{i}$. We don't repeat the definition here. These will be called "parabolic bundles" for short. If $E$ is a parabolic bundle then for each $q_{i}$ and each $\alpha \in \mathbb{R}$ we have an associated graded vector space $G r_{\alpha, q_{i}}(E)$ which is finite-dimensional. Multiplication by a local coordinate at $q_{i}$ gives an isomorphism

$$
G r_{\alpha, q_{i}}(E) \cong G r_{\alpha+1, q_{i}}(E) .
$$

Define the residue of $E$ at $p_{i}$ to be the associated-graded direct sum

$$
\operatorname{res}\left(E ; q_{i}\right):=\bigoplus_{0 \leq \alpha<1} G r_{\alpha, q_{i}}(E)
$$

For a fixed $\lambda \in \mathbb{C}$ there is a notion of logarithmic $\lambda$-connection $\nabla$ on a parabolic bundle $E$. The logarithmic structure is with respect to the divisor $Q=q_{1}+\ldots+$ $q_{n}$. For any $\alpha_{1}, \ldots, \alpha_{n}$ it induces a $\lambda$-connection

$$
\nabla: E_{\alpha_{1}, \ldots, \alpha_{n}} \rightarrow E_{\alpha_{1}, \ldots, \alpha_{n}} \otimes \Omega_{Z}^{1}(\log Q) .
$$

With the canonical isomorphism $\Omega_{Z}^{1}(\log Q)_{q_{i}} \cong \mathbb{C}$, a $\lambda$-connection on the parabolic bundle $E$ induces an endomorphism called the residue

$$
\operatorname{res}\left(\nabla ; q_{i}\right): \operatorname{res}\left(E ; q_{i}\right) \rightarrow \operatorname{res}\left(E ; q_{i}\right) .
$$

The residue of the pair $(E, \nabla)$ at a point $q_{i}$ is defined as the pair of an $S^{1}$-graded vector space with endomorphism

$$
\operatorname{res}\left(E, \nabla ; q_{i}\right):=\left(\operatorname{res}\left(E ; q_{i}\right), \operatorname{res}\left(\nabla ; q_{i}\right)\right) .
$$

When necessary, we introduce an index to denote the piece $\operatorname{res}\left(\nabla ; q_{i}\right)_{\alpha}$ acting on $G r_{\alpha, q_{i}}(E) \subset \operatorname{res}\left(E ; q_{i}\right)$.

If $F \subset E$ is a parabolic subbundle compatible with $\nabla$ then we can consider its parabolic degree, and the parabolic slope is the parabolic degree divided by the rank. We say that $(E, \nabla)$ is stable (resp. semistable) if for any strict parabolic subbundle compatible with $\nabla$, the parabolic slope of $F$ is strictly less than (resp. less than or equal to) that of $E$.

Given a parabolic bundle $E$, define its parabolic type at $q_{i}$ to be the divisor on $S^{1}$

$$
\operatorname{type}\left(E, q_{i}\right):=\sum_{0 \leq \alpha<1}\left(\operatorname{dim} G r_{\alpha, q_{i}}(E)\right) \cdot[\alpha]
$$


This follows the discussion in $\S 4.5$ with $\mathbb{L}=S^{1}$.

Up to isomorphism, the residue at $q_{i}$ of a parabolic logarithmic $\lambda$-connection $\operatorname{res}\left(E, \nabla ; q_{i}\right)$ is classified by specifying its type $\sum g_{\alpha}[\alpha]$ and for each $\alpha$, specifying the Jordan normal form of an endomorphism of a vector space of dimension $g_{\alpha}$.

We say that the residues of $(E, \nabla)$ are semisimple if the Jordan normal forms are diagonalizable. This means that on each $G r_{\alpha, q_{i}}$ the action of $\operatorname{res}\left(\nabla ; q_{i}\right)_{\alpha}$ is semisimple or diagonalizable, so it corresponds to a divisor on $\mathbb{A}^{1}$. Altogether, when the residues are semisimple, the isomorphism class of the residue of $(E, \nabla)$ at $q_{i}$ is determined by a divisor of total degree $r$ on $S^{1} \times \mathbb{A}^{1}$.

Recall that we have a notion of tame harmonic bundle on $Z-Q$. A harmonic bundle consists of a flat connection, and an equivariant harmonic map. The tameness condition means that locally near the singularities, the the flat sections of the connection on sectors have polynomial growth with respect to the harmonic metric. Measuring the growth rate of sections leads to parabolic structures. The flat connection decomposes as

$$
D=D^{\prime}+D^{\prime \prime}=(\partial+\bar{\theta})+(\bar{\partial}+\theta)
$$

where $\partial+\bar{\partial}$ is a unitary connection, $\theta+\bar{\theta}$ antipreserves the metric, and $\theta$ is holomorphic. Fix $\lambda \in \mathbb{C}$ which allows us to define a differential $\lambda$-connection

$$
D_{\lambda}:=\lambda D^{\prime}+D^{\prime \prime} .
$$

The $(0,1)$ piece, which has contributions from both terms $\lambda D^{\prime}$ and $D^{\prime \prime}$, is a usual holomorphic structure giving rise to a holomorphic vector bundle $E$. The $(1,0)$ piece is a holomorphic $\lambda$-connection on $E$.

Measuring the growth rate of sections in a holomorphic frame, leads to an extension of $E$ as a parabolic bundle over $Z$ with parabolic structure along $Q$, again denoted $E$. The connection $\nabla=\left(D_{\lambda}\right)^{1,0}$ is a logarithmic $\lambda$-connection on the parabolic bundle $E$. The parabolic logarithmic $\lambda$-connection $(E, \nabla)$ is polystable, in other words a direct sum of stable objects of the same slope.

Conversely, given a parabolic logarithmic $\lambda$-connection $(E, \nabla)$, if it is polystable then there exists an essentially unique structure of harmonic bundle given by a harmonic metric on $E$ over $X-Q$ with the appropriate growth rates determined by the parabolic structure. The metric connection is unique and the metric is unique up to a positive real scalar on each stable piece. The Higgs case is $\lambda=0$. 
In keeping with Convention 2.1, we would like to insure that the monodromy transformations are semisimple. We furthermore assume that the residues of $\theta$ vanish. This amounts to restricting to representations where the local monodromy eigenvalues are in $S^{1} \subset \mathbb{G}_{m}$ together with trivial filtered local system structures at the singularities.

Convention 6.1. The residue of the Higgs field $\theta$ on the associated-graded of the parabolic bundle $G r_{\alpha, q_{i}}(E)$ at any point $q_{i} \in Q$ is equal to zero.

Lemma 6.2. If $(E, \theta)$ is a polystable parabolic Higgs bundle of degree zero satisfying Convention 6.1 then the monodromy transformations of the corresponding local system are semisimple, with eigenvalues in $S^{1}$ corresponding to $e^{2 \pi i \alpha}$ for $\alpha$ the parabolic weights.

Proof: This follows from the local considerations shown in [129].

The moduli space of parabolic logarithmic $\lambda$-connections can be constructed, see many references on parabolic bundles included in the bibliography below. This moduli space becomes isomorphic (as a real analytic space possibly with singularities) to a space of harmonic bundles which can be constructed as in Hitchin's original case [70], see also Fujiki [54]. As $\lambda$ varies we get a family over the affine line, which is the nonabelian Hodge filtration on the moduli space. Glueing with the complex conjugate we get the twistor space for Hitchin's hyperkähler structure [70] [54]. In our case of an open curve, some further work is needed, see [114] for example. We should deal with the transformation of residual types which occurs when we change $\lambda$ [129], a situation which appears to reflect some kind of weight-two phenomenon corresponding to the punctures. In the case of quasiunipotent monodromy we should be able to deal with the problem by looking at local systems with unipotent monodromy on a DM-curve. This general moduli problem will not be considered any more here, but constitutes a good source of further questions: first and foremost we would like to have Katz isomorphisms between these moduli spaces coming from a parabolic middle convolution.

6.1. Cyclotomic harmonic bundles. Our construction of Higgs bundles will be based on a trick to insure stability. Recall that $\mathbb{C}^{*}$ acts on the space of parabolic Higgs bundles by $t:(E, \theta) \mapsto(E, t \theta)$. If we assume that the residue of $\theta$ is unipotent (or even equal to zero if we want to keep with Convention 2.1) then this action preserves the residue of the parabolic structure and $\theta$, so by [129] 
it preserves the local type of monodromy transformations of the corresponding local system.

Recall that a complex variation of Hodge structure is a harmonic bundle which is a fixed point for the full action of $\mathbb{C}^{*}$ or equivalently for the action of $S^{1}$. A variant is to look at the action of a finite cyclic subgroup of roots of unity $\mu_{m} \subset \mathbb{C}^{*}$. Recall [70] [30] that the action of $S^{1}$ preserves the harmonic metric structure. A harmonic bundle which is a fixed point of the action of $\mu_{m}$ will be called a cyclotomic harmonic bundle, where $m \geq 2$ is considered as fixed for now-later we can say " $m$-cyclotomic" if we need to specify $m$.

When we say that $(E, \theta)$ is a fixed point this means that it is provided with an additional structure of an action of $\mu_{m}$ on $E$ such that for any $u \in \mu_{m}$ and $e \in E$ we have $(u \theta)(u e)=u(\theta e)$.

The structure of a cyclotomic harmonic bundle is very similar to the structure of a complex variation of Hodge structure. The group of characters of $\mu_{m}$ is canonically isomorphic to $\mathbb{Z} / m \mathbb{Z}$ because we have defined $\mu_{m}$ as coming from a privileged embedding $\mu_{m} \subset \mathbb{C}^{*}$. The action of $\mu_{m}$ on $E$ provides a decomposition according to characters

$$
E=\bigoplus_{p \in \mathbb{Z} / m \mathbb{Z}} E^{p}
$$

and the formula $(u \theta)(u e)=u(\theta e)$ then says

$$
\theta: E^{p} \rightarrow E^{p+1} \otimes \Omega_{Z}^{1}(\log Q) .
$$

The only difference with the case of variations of Hodge structure is that $p+1$ is taken in the quotient group $\mathbb{Z} / m \mathbb{Z}$ so $\theta$ includes a piece of the form

$$
\theta: E^{m} \rightarrow E^{1} \otimes \Omega_{Z}^{1}(\log Q) .
$$

Thus, $\theta$ is no longer required to be a nilpotent transformation. In the parabolic case, the decomposition is compatible with the parabolic structure.

The action of $\mu_{m} \subset S^{1}$ preserves the differential operators of the harmonic bundle, so by averaging we can always choose a $\mu_{m}$-invariant harmonic metric. The decomposition of $E$ is then orthogonal and the pieces $E^{p}$ are preserved by the metric connection $\partial+\bar{\partial}$. The complex conjugate $\bar{\theta}$ goes from $E^{p}$ to $A^{0,1}\left(E^{p-1}\right)$. Thus, in all respects this looks like a complex variation of Hodge structure except that the Hodge decomposition is viewed circularly and the Kodaira-Spencer components can go all the way around the circle. 
Hitchin in [72] gave a construction of a subspace of Higgs bundles which corresponded to an analogue of Teichmüller space. In Hitchin's construction, a basic variation of Hodge structure is modified by adding a new term in the Higgs field. In this sense, the notion of cyclotomic Higgs field is a variant on [72]. Hitchin's Teichmüller Higgs bundles can sometimes be cyclotomic: in the notation of [72] if $\alpha_{m} \neq 0$ but $\alpha_{m-1}=\ldots=\alpha_{2}=0$ then the Higgs field written down there is cyclotomic.

The correspondence between Higgs bundles and local systems is compatible with the action of $\mu_{m}$, and this helps with the stability condition. Suppose $(E, \theta)$ is an $m$-cyclotomic Higgs bundle, that is a bundle with action of $\mu_{m}$ (or equivalently a decomposition as above) compatible with the action on $\theta$. We say that it is cyclotomically semistable (resp. cyclotomically stable) if for any $\mu_{m^{-}}$ invariant sub-Higgs bundle, the slope is smaller (resp. strictly smaller) than the slope of $E$.

Proposition 6.3. Suppose $(E, \theta)$ is a cyclotomically stable parabolic cyclotomic Higgs bundle of parabolic degree zero. Then $(E, \theta)$ is polystable as a regular parabolic Higgs bundle, and it has a $\mu_{m}$-equivariant harmonic metric with growth rates corresponding to the parabolic structure.

Proof: The $\beta$-subbundle is $\mu_{m}$-invariant, so cyclotomic semistability implies semistability. The socle is $\mu_{m}$-invariant, so cyclotomic polystability implies polystability. Then [128], Theorem 1 which took into account the possibility of having the action of a group such as $\mu_{m}$, provides an invariant harmonic metric. The growth rates are governed by the choice of initial metric, as discussed in [128] $\$ 10$ and [129].

It is interesting to note that the cyclotomic Higgs bundles play a special role in the compactification of the de Rham moduli space. Recall that the compactification puts at infinity a divisor obtained by dividing $M_{\text {Higgs }}-\{\theta$ nilpotent $\}$ by the action of $\mathbb{C}^{*}$. If we take the quotient in the sense of stacks, then the compactification becomes a DM stack and the stacky points with automorphism group $\mu_{m}$ are exactly the cyclotomic Higgs bundles.

6.2. The maximal case. Traditionally one of the easiest cases is when the $E^{p}$ are line bundles. For this, take $m=r$ equal to the rank. 
Lemma 6.4. Suppose $E=\bigoplus E^{p}$ is an $r$-cyclotomic parabolic Higgs bundle of rank $r$ with $\theta$ not nilpotent. This means that the $E^{p}$ are parabolic line bundles and every component $\theta^{p}: E^{p} \rightarrow E^{p+1} \otimes \Omega_{Z}^{1}$ is nonzero. Then $(E, \theta)$ is cyclotomically stable, hence polystable as an ordinary parabolic Higgs bundle.

Proof: Non-nilpotence of $\theta$ requires that all the components be nonzero, in particular all of the bundles $E^{p}$ are nonzero. Since their number is equal to the rank, they must be line bundles. Suppose $F \subset E$ is a $\mu_{r}$-invariant saturated subbundle. It decomposes as a direct sum of $F^{p} \subset E^{p}$. If any one of the $F^{p}$ is nonzero then all of them are nonzero because every component $\theta^{p}$ is nonzero and they go around in a circle. If $F$ is saturated we get $F^{p}=E^{p}$. Thus, there are no $\mu_{r}$-invariant subbundles of rank strictly between 0 and $r$, so $E$ is vacuously cyclotomically stable. By Proposition $6.3,(E, \theta)$ is polystable in the ordinary sense.

The structure of a non-nilpotent $r$-cyclotomic parabolic Higgs bundle is particularly easy to understand. It consists of a collection of parabolic line bundles $E^{1}, \ldots, E^{r}$, together with morphisms of parabolic bundles

$$
\theta^{p}: E^{p} \rightarrow E^{p+1} \otimes \Omega_{Z}^{1}(\log Q) .
$$

Convention 6.1 says that we want $\operatorname{res}\left(\theta^{p}, q_{i}\right)$ to induce the zero map on the associated-graded $G r_{\alpha, q_{i}}$ for any $\alpha$ and any singular point $q_{i} \in Q$. In view of the fact that we are dealing with parabolic line bundles, there is only one weight on each side, and the residue map is automatically zero unless the two weights are the same. This will appear in our criterion below.

6.3. Explicit construction. A parabolic line bundle on $(Y, Q)$ is always of the form

$$
E=\mathcal{O}_{Y}(k)\left(a_{1} q_{1}+\ldots+a_{n} q_{n}\right)
$$

with $k \in \mathbb{Z}$ and $a_{i} \in[0,1)$. Written in this way, the vector $\left[k ; a_{1}, \ldots, a_{n}\right]$ is uniquely determined and uniquely determines $E$, and we may use it as notation. Suppose we are given two parabolic line bundles $E=\left[k ; a_{1}, \ldots, a_{n}\right]$ and $E^{\prime}=$ $\left[k^{\prime} ; a_{1}^{\prime}, \ldots, a_{n}^{\prime}\right]$. A map from $E$ to $E^{\prime}$ will consist of a holomorphic map

$$
f: \mathcal{O}_{Y}(k) \rightarrow \mathcal{O}_{Y}\left(k^{\prime}\right)
$$

such that if $a_{i}>a_{i}^{\prime}$ then $f\left(q_{i}\right)=0$. In this description we use the fact that $a_{i}, a_{i}^{\prime} \in[0,1)$, in particular $\left|a_{i}-a_{i}^{\prime}\right|<1$ always. 
In order to have a map which furthermore induces the zero map on associated graded spaces at each $q_{i}$,

$$
G r_{\alpha, q_{i}}(f)=0: G r_{\alpha, q_{i}}(E) \rightarrow G r_{\alpha, q_{i}}\left(E^{\prime}\right),
$$

we should require that $f\left(q_{i}\right)=0$ also when $a_{i}=a_{i}^{\prime}$. Thus, the description of these maps (which we call zero-residue maps) is that $f\left(q_{i}\right)=0$ when $a_{i} \geq a_{i}^{\prime}$.

Finally, we get to a description of a map

$$
f: E \rightarrow E^{\prime} \otimes \Omega_{Y}^{1}(\log Q)
$$

inducing zero on the residues as required by Convention 6.1 . Recall that $\Omega_{Y}^{1}(\log Q)=$ $\mathcal{O}_{Y}(n-2)$. Thus, such a map $f$ is the same thing as a holomorphic section

$$
f \in \Gamma\left(Y, \mathcal{O}_{Y}\left(k^{\prime}-k+n-2\right)\right)
$$

such that $f\left(q_{i}\right)=0$ whenever $a_{i} \geq a_{i}^{\prime}$, or we can also say

$$
f \in \Gamma\left(Y, \mathcal{O}_{Y}\left(k^{\prime}-k+n-2-\#\left\{i, a_{i} \geq a_{i}^{\prime}\right\}\right)\right) .
$$

We get the following lemma.

Lemma 6.5. Suppose $E=\left[k ; a_{1}, \ldots, a_{n}\right]$ and $E^{\prime}=\left[k^{\prime} ; a_{1}^{\prime}, \ldots, a_{n}^{\prime}\right]$ are parabolic line bundles on $(Y, Q)$. Then there exists a nontrivial zero-residue map

$$
f: E \rightarrow E^{\prime} \otimes \Omega_{Y}^{1}(\log Q)
$$

if and only if

$$
\#\left\{i, a_{i} \geq a_{i}^{\prime}\right\} \leq k^{\prime}-k+n-2 .
$$

If equality holds then the map $f$ has no zeroes other than as required for the residues, and is unique up to a scalar constant. If the inequality is strict then $f$ has other zeroes.

Suppose we are given a vector of divisors $\vec{g}=\left(g_{1}, \ldots, g_{n}\right) \in \operatorname{Div}\left(S^{1}\right) n$, with $\operatorname{deg}\left(g_{i}\right)=r$. Choose the standard section of $\mathbb{R} \rightarrow \mathbb{R} / \mathbb{Z}$ to identify $S^{1} \cong[0,1)$. Write

$$
g_{i}=\sum_{\alpha \in[0,1)} m_{i}(\alpha)[\alpha]
$$

A sequence $a_{i, 1}, a_{i, 2}, \ldots, a_{i, r} \in[0,1)$ is called an arrangement of $g_{i}$ if each $\alpha$ occurs in the sequence with multiplicity $m_{i}(\alpha)$. This is to say that the sequence 
of exponentials of the $a_{i, j}$ is a possible sequence of eigenvalues along the diagonal, for a matrix in the conjugacy class $C\left(g_{i}\right)$.

An arrangement is called good if the number of indices $t$ with $a_{i, t} \geq a_{i, t+1}$ is minimal. Let $T\left(g_{i}\right)$ be the minimal number of such indices, thus the arrangement is good if

$$
\#\left\{t, a_{i, t} \geq a_{i, t+1}\right\}=T\left(g_{i}\right) .
$$

Here, and always below, the indices are taken modulo $r$, for example if $t=r$ then $a_{i, t+1}=a_{i, 1}$. Enumerate the indices $t$ as above, in increasing order $t_{1}<\ldots<t_{p}$. We can thus write our arrangement as a "sawtooth":

$$
\begin{gathered}
a_{i, 1}<a_{i, 2}<\ldots<a_{i, t_{1}} \\
a_{i, t_{1}} \geq a_{i, t_{1}+1} \\
a_{i, t_{1}+1}<\ldots<a_{i, t_{2}} \\
a_{i, t_{2}} \geq a_{i, t_{2}+1} \\
\vdots \\
a_{i, t_{p}} \geq a_{i, t_{p}+1} \\
a_{i, t_{p}+1}<\ldots<a_{i, r}
\end{gathered}
$$

with $a_{i, r}<a_{i, 1}$ unless $t_{p}$ happens to be $r$. Now let

$$
g_{i, j}:=\left[a_{i, t_{j}+1}\right]+\left[a_{i, t_{j}+2}\right]+\ldots+\left[a_{i, t_{j+1}-1}\right]+\left[a_{i, t_{j+1}}\right],
$$

with $t_{j+1}:=t_{1}$ when $j=p$ and the terms in $g_{i, p}$ adapted appropriately. These are reduced effective divisors, that is each eigenvalue occurs with multiplicity at most 1 , because the sequences are strictly increasing in between the $t_{j}$. And we have a decomposition

$$
g_{i}=g_{i, 1}+\ldots+g_{i, p}
$$

Notice that

$$
t_{j+1}=\operatorname{deg}\left(g_{i, j}\right)+t_{j} \quad(\text { modulo } r) \text {. }
$$

Conversely, given a pair of $p$-uples written $\left(t_{1}, \ldots, t_{p} ; g_{i, 1}, \ldots, g_{i, p}\right)$ with $t_{j}$ an increasing sequence in $1, \ldots, r$ and the $g_{i, j}$ giving a decomposition of $g_{i}$ into reduced effective divisors, we get an arrangement. The arrangement is good if $p$ is minimal and equal to the maximal multiplicity in $g_{i}$. There is a one-to-one correspondence between such notations and arrangements for $g_{i}$.

Lemma 6.6. The minimal number of $t$ 's is equal to the maximum multiplicity in the divisor $g_{i}$,

$$
T\left(g_{i}\right)=\max _{\alpha} m_{i}(\alpha)
$$


Proof: It is easy to see that for any $\alpha$ we have $T\left(g_{i}\right) \geq m_{i}(\alpha)$. On the other hand, we can clearly choose a decomposition into reduced effective divisors $g_{i}=$ $g_{i, 1}+\ldots+g_{i, p}$ with $p=\max _{\alpha} m_{i}(\alpha)$. Thus the minimal $p$ is equal to the maximum of the $m_{i}(\alpha)$.

Now suppose that for each $i=1, \ldots, g$ we have chosen a good arrangement $a_{i, j}$ for $g_{i}$. Let

$$
\left(t_{i, 1}, \ldots, t_{i, p_{i}} ; g_{i, 1}, \ldots, g_{i, p_{i}}\right)
$$

be the notation established above with $p_{i}=T\left(g_{i}\right)$. For any sequence $k_{1}, \ldots, k_{r}$ define parabolic line bundles

$$
E^{j}:=\left[k_{j} ; a_{1, j}, \ldots, a_{n, j}\right] .
$$

In order to construct a cyclotomic Higgs bundle $E=\bigoplus_{j=1}^{r} E^{j}$, we investigate the possible choice of $k_{1}, \ldots, k_{r}$ such that there are nontrivial zero-residue maps

$$
\theta^{j}: E^{j} \rightarrow E^{j+1} \otimes \Omega_{Y}^{1}(\log Q)
$$

including the case $j=r, j+1=1$. Let $\tau_{j}$ denote the cardinality

$$
\tau_{j}:=\#\left\{i, \quad a_{i, j} \geq a_{i, j+1}\right\} .
$$

For any sequence of $k_{j}$ put

$$
z_{j}:=k_{j+1}-\left(\tau_{j}+k_{j}+2-n\right) .
$$

Lemma 6.7. With the above notations, there exist zero-residue maps $\theta^{j}$ if and only if $z_{j} \geq 0$ for $j=1, \ldots, r$. In this case, $z_{j}$ is the number of extra zeros of $\theta^{j}$ beyond what are required by the zero-residue condition. The $z_{j}$ are subject to the relation

$$
z_{1}+\ldots+z_{r}=\delta(\vec{g}),
$$

so there exists a possible choice of $z_{j}$ or equivalently of $k_{j}$ if and only if $\delta(\vec{g}) \geq 0$.

Proof: The first statements come from Lemma 6.5. From the definition of $T\left(g_{i}\right)$ and $\tau_{j}$ we get

$$
\sum_{j=1}^{r} \tau_{j}=\sum_{i=1}^{n} T\left(g_{i}\right)
$$

Thus

$$
z_{1}+\ldots+z_{r}=r(n-2)-\sum_{i=1}^{n} T\left(g_{i}\right)=\delta(\vec{g}) .
$$


Given $z_{j} \geq 0$ subject to the relation $z_{1}+\ldots+z_{r}=\delta(\vec{g})$, and given $k_{1}$, we obtain the remaining $k_{2}, \ldots k_{r}$ from the formula for $z_{j}$. Construct the parabolic bundles $E^{j}$ and nontrivial zero-residue maps $\theta^{j}$. This yields an $r$-cyclotomic Higgs bundle $(E, \theta)$.

6.4. The degree. As pointed out in Lemma 6.4, the $(E, \theta)$ constructed this way is cyclotomically stable, hence polystable as a Higgs bundle. To finish the construction of a local system we need to insure that its parabolic degree vanishes. The parabolic degree of $E^{j}=\left[k_{j} ; a_{1, j}, \ldots, a_{n, j}\right]$ is

$$
\operatorname{deg}^{\operatorname{par}}\left(E^{j}\right)=k+a_{1, j}+\ldots+a_{n, j} .
$$

Adding up gives the parabolic degree of $E$ :

$$
\operatorname{deg}^{\operatorname{par}}(E)=\sum_{j=1}^{r} k_{j}+\sum_{i, j} a_{i, j}=\sum_{j=1}^{r} k_{j}+\sum_{i, \alpha} m_{i}(\alpha) \alpha .
$$

By induction,

$$
k_{j+1}=k_{1}+z_{1}+\ldots+z_{j}+\tau_{1}+\ldots+\tau_{j}+j(2-n) .
$$

We have

$$
\operatorname{deg}^{\text {par }}(E)=P+k_{1} r+\sum_{j=1}^{r}(r-j)\left(z_{j}+\tau_{j}\right)
$$

where

$$
P:=\sum_{i, \alpha} m_{i}(\alpha) \alpha+\sum_{j=1}^{r} j(r-j)(2-n)
$$

represents the piece which doesn't depend on the choice of arrangements or of $k_{j}$. The condition $\operatorname{Det}(\vec{g})=1$ says that $P \in \mathbb{Z}$.

Recall that $\tau_{j}$ is the number of $i$ such that $a_{i, j} \geq a_{i, j+1}$. This is the same as the number of $i$ such that $j \in\left\{t_{i, 1}, \ldots, t_{i, p_{i}}\right\}$. The terms involving $\tau_{j}$ can be recast as a sum over the elements $t_{i, j}$. We conclude the following formula for the parabolic degree of the Higgs bundle we have constructed:

$$
\operatorname{deg}^{\operatorname{par}}(E)=P+k_{1} r+\sum_{i=1}^{n} \sum_{j=1}^{p_{i}}\left(r-t_{i, j}\right)+\sum_{j=1}^{r}(r-j) z_{j} .
$$


Theorem 6.8. Suppose given a vector of divisors $\vec{g} \in \operatorname{Div}\left(S^{1}\right)^{n}$ such that $\operatorname{Det}(\vec{g})=1$. Suppose that the defect is strictly positive,

$$
r(n-2)-\sum T\left(g_{i}\right)=\delta(\vec{g})>0 .
$$

Fix any collection of good arrangements $a_{i, j}$ for $\vec{g}$. Then it is possible to choose the $k_{j}$ subject to the constraint

$$
k_{j+1} \geq \tau_{j}+k_{j}+2-n,
$$

such that $\operatorname{deg}^{\mathrm{par}}(E)=0$.

Proof: Fixing the collection of good arrangements, the terms in (6.2) involving $t_{i, j}$ are fixed. Because of the strictly positive defect, there is a nontrivial choice of $z_{1}, \ldots, z_{j}$. Geometrically this means that we have a choice as to how many zeros $\theta_{j}$ can have.

Put $z_{r}:=\delta(\vec{g})-1$ and $z_{j}=0$ for all but one value of $j=j^{\prime}$ in which case $z_{j^{\prime}}=1$. Make the convention here that if $j^{\prime}=r$ then $z_{r}:=\delta(\vec{g})$ instead. With this choice we get

$$
\sum_{j=1}^{r}(r-j) z_{j}=r-j^{\prime},
$$

and by choosing $j^{\prime}$ appropriately this can take on any value between 0 and $r-1$. In particular, modulo $r$ it can take on all values. By adjusting $k_{1}$ appropriately, $\operatorname{deg}^{\operatorname{par}}(E)$ can take on any integer value.

Theorem 6.9. Suppose given a vector of divisors $\vec{g} \in \operatorname{Div}\left(S^{1}\right)^{n}$ such that $\operatorname{Det}(\vec{g})=1$. Suppose that the defect is zero,

$$
r(n-2)-\sum T\left(g_{i}\right)=\delta(\vec{g})=0,
$$

but the superdefect is strictly positive $\sigma(\vec{g})>0$. Then it is possible to choose a good arrangement $\left\{a_{i, j}\right\}$ for $\vec{g}$ and $k_{1}$, which determines the remaining $k_{j}$ in the zero-defect case by the constraint

$$
k_{j+1}=\tau_{j}+k_{j}+2-n,
$$

such that $\operatorname{deg}^{\mathrm{par}}(E)=0$. 
Proof: In the case where the defect is zero, we are constrained to have $z_{j}=0$. In particular, once we fix $k_{1}$ then the others are determined. We have the simplified formula

$$
\operatorname{deg}^{\text {par }}(E)=P+k_{1} r+\sum_{i=1}^{n} \sum_{j=1}^{p_{i}}\left(r-t_{i, j}\right) .
$$

On the other hand, the fact that the superdefect is nonzero means that for some $i$ there is at least one eigenvalue $\alpha^{\prime}$ which appears with multiplicity $m_{i}\left(\alpha^{\prime}\right)<p_{i}=$ $\max _{\alpha} m_{i}(\alpha)$. In particular, for any arrangement which we denote now generically by $A$, we have at least one interval not containing the eigenvalue $\alpha^{\prime}$. Define the following operation on arrangements: find an interval $t_{i, j}+1, \ldots, t_{i, j+1}$ containing $\alpha^{\prime}$ but such that the preceding interval $t_{i, j-1}+1, \ldots, t_{i, j}$ doesn't contain $\alpha^{\prime}$. Move $\alpha^{\prime}$ from the one to the other. We get a new arrangement $\partial A$ with the property that all $t_{i^{\prime}, j^{\prime}}(\partial A)$ are the same as for $A$, except

$$
t_{i, j}(\partial A)=t_{i, j}(A)+1 .
$$

Note that $\partial A$ will always be a good arrangement whenever $A$ is good. From this and the above formula we find

$$
\operatorname{deg}^{\operatorname{par}}(E(\partial A))=\operatorname{deg}^{\text {par }}(E(A))-1 .
$$

In particular, iterating the operation $A \mapsto \partial A$ and modifying $k_{1}$ we find that $\operatorname{deg}^{\text {par }}(E(A))$ can take on all integer values as $A$ runs through all the good arrangements.

Putting together these two theorems we get:

Corollary 6.10. Suppose $\vec{g} \in \operatorname{Div}\left(S^{1}\right)^{n}$ is a vector of local monodromy data with eigenvalues in $S^{1} \subset \mathbb{G}_{m}$. Suppose the defect is positive $\delta(\vec{g}) \geq 0$. If $\delta=0$ then suppose that the superdefect is strictly positive; this is equivalent to supposing that the virtual dimension of the moduli space is at least 4 . Then there exists a parabolic $r$-cyclotomic Higgs bundle $(E, \theta)$ of parabolic degree 0, cyclotomically stable and polystable in the usual sense, corresponding to a local system with local monodromy data $\vec{g}$.

The only cases left to be treated are when the moduli space has dimension 2 . There are four families as listed in Lemma 2.13. These cases are considered by Kostov in [90]. As he notes there, the determinant of the vector $\frac{\vec{g}}{d}$ is a $d$-th root of unity. For $d>1$ and primitive root of unity, it looks like there cannot be an 
r-cyclotomic Higgs bundle; however a solution exists [92] and one might hope to construct an $m$-cyclotomic Higgs bundle for smaller $m$ and with some component bundles $E^{j}$ of rank 2 . When the root of unity is not primitive, Kostov shows that there are no irreducible solutions.

\section{Further QUeSTIONS}

It would be good to have the full middle-convolution theory for the general setup of parabolic logarithmic $\lambda$-connections [110] [111] [112] [125]. This raises some nontrivial questions such as defining the middle higher direct image in the parabolic setting, obtaining a base-change result analogous to Convention 5.3, and showing polystability of the middle convolution. It was my original goal to treat these questions here but that turned out to be very difficult.

Aker and Szabo have communicated to me their recent preprint [3] in which they do the Nahm transform (essentially the same as Fourier transform) for parabolic Higgs bundles with irregular singularities having poles of order $\leq 2$ at infinity. This should allow one to obtain the middle convolution for parabolic Higgs bundles by following Katz's original method.

If the weights of a parabolic structure are rational, i.e. for every point $q_{i}$ the parabolic type is a divisor concentrated over roots of unity in $S^{1}$, then as discussed in [19] [27] [108] [77], the parabolic bundle may also be viewed as a bundle on a Deligne-Mumford stack $Z\left[\frac{Q}{m}\right]$ obtained by assigning an integer $m$ to the points $q_{i}$. Here $m$ should be chosen to be divisible by all the denominators of the rational weights which occur. In this case, a logarithmic $\lambda$-connection on the parabolic bundle may also be viewed as a logarithmic connection on the corresponding DMbundle. An intermediate case between the non-parabolic case we have discussed in $\S 5$ and the general case of parabolic logarithmic $\lambda$-connections, would be the case of parabolic logarithmic $\lambda$-connections with rational weights. Also assuming that the residue of the connection on $G r_{\alpha, q_{i}}(E)$ is the scalar $\alpha$, these objects would be equivalent to local systems on the DM-stacks $Z\left[\frac{Q}{m}\right]$. It should be possible to have a theory of Katz's middle convolution for these objects. The blown-up surface $(X, J)$ would be provided with a stack structure and the singular fibers would be twisted curves [1] [2].

The moduli spaces have numerous additional structures. 
Conjecture 7.1. The isomorphisms between different moduli spaces given by the middle convolution map, preserve the Hodge filtration, the $\mathbb{C}^{*}$ action, and the Hitchin hyperkähler structure when this is defined, that is when the eigenvalues of the local monodromy transformations are in $S^{1}$.

This conjecture can probably be proven by Aker and Szabo with their method [3], indeed they show that the Nahm transform preserves the hyperkähler structure of the moduli spaces.

Völklein points out in [139] that the Katz isomorphisms between various Betti moduli spaces are compatible with the action of the braid group of the points $q_{1}, \ldots, q_{n} \in \mathbb{P}^{1}$. Similarly, the cohomological formulation immediately implies that for $n \geq 4$ the Katz isomorphisms between different de Rham moduli spaces are compatible with the nonabelian Gauss-Manin connection, i.e. the isomonodromic deformation equations. This was used by Boalch to get information about finite Painlevé orbits in [14] [15]. It would be interesting to look further at the dynamics of the braid action and the isomonodromy equations.

Question 7.2. Which Hodge types can occur at variations of Hodge structure in the moduli spaces? How does the Hodge type change under middle convolution?

To what extent do we get unexpected or exceptional automorphisms of moduli spaces, due to the possibility of running Katz's algorithm in several different ways? In particular, one could start in the range $\delta \geq 0$, do a series of middle convolutions which go out of this range, then another series to go back. In some cases this should change the local monodromy vector, so we should obtain isomorphisms $M_{B}\left(\vec{g}_{1}\right) \cong M_{B}\left(\vec{g}_{2}\right)$ for $g_{1} \neq g_{2}$ in the range $\delta \geq 0$. Say that these two local monodromy vectors are middle-convolution equivalent in this case.

Question 7.3. What is the quotient of the set of local monodromy vectors with $\delta \geq 0$, by the relation of middle-convolution equivalence? In each middle convolution equivalence class, does the operation of going out and back again provide any nontrivial automorphisms of $M_{B}$ ?

Question 7.4. Is there a Torelli theorem saying that the isomorphism class of $M_{B}(\vec{g})$ and/or $M_{D R}(\vec{g})$ possibly with additional structures such as the Hodge filtration, the hyperkähler metric, etc., determines the middle convolution equivalence class of $\vec{g}$ (and maybe the collection of points $Q$ depending on how much structure we are considering)? 
Problem 7.5. Generalize Roberts' observations on the geography of the Katz algorithm [123] to the nonrigid case.

It would be good to compare explicitly what is happening in our presentation, which basically follows Kostov's notation and setup, with the notation and setup used by Crawley-Boevey. Note that in Crawley-Boevey's point of view, the Katz operations are root reflections, and he uses several reflections in a row to get into a positive Weyl chamber before giving an explicit construction. This is obviously basically the same procedure as what we are doing here. It would be good to compare the numbers, and also to recover Roberts' results and observations [123] in the Crawley-Boevey formulation.

What is the exact relationship between our de Rham version of the middle convolution, and the algebraic operations on Fuchsian systems considered by Kostov, Haraoka-Yokoyama, Crawley-Boevey?

Theorem 2.9 says that the middle cohomology of $\operatorname{End}(E)$ gives the deformation and obstruction theory for the moduli space of representations with fixed conjugacy classes on a curve. Remembering that the middle cohomology is really intersection cohomology, this suggests that we should ask for the geometric interpretation of the intersection cohomology of $\operatorname{End}(E)$ in the higher dimensional case. More precisely, is there a natural derived moduli stack of local systems generalizing Kapranov [80] based on the intersection cohomology? And, what kind of geometric objects does this derived moduli stack parametrize?

7.1. Low-dimensional cases. One of the main reasons for looking at moduli spaces of representations on the punctured Riemann sphere is that these give many more examples with small dimension, than are obtained from Hitchin's original case of compact Riemann surfaces. This was first pointed out by Hausel [65] with his "toy example". We have constructed local systems whenever the virtual dimension is $\geq 4$. In some sense the first case to look at is dimension 2, which has to be one of the cases listed in Lemma 2.13. Unfortunately, our technique of construction broke down in this case, but we can hope to have a variant.

The explicit techniques applied by Gleizer in the rigid case [55] should be applicable to low-dimensional cases. 
It would be interesting to compute as explicitly as possible all of the various structures and properties for some concrete low-dimensional cases. For example, what does the $\mathbb{C}^{*}$ action on the moduli space look like? Some things to study in low-dimensional cases would be: compactifications [65] and their the dynamics [37] [38] [39] [12], the Hitchin system [70] [66], the relationship with Painlevé equations [71] [14] [15] [47], jumps and wall-crossing phenomena such as in [134] [114], real structures and Toledo invariants [24] [26] [106] [140].

\section{REFERENCES}

[1] D. Abramovich, A. Vistoli. Compactifying the space of stable maps. J. Amer. Math. Soc. 15 (2002), 27-75.

[2] D. Abramovich, T. Graber, A. Vistoli. Algebraic orbifold quantum products, Orbifolds in mathematics and physics (Madison, WI, 2001), Contemp. Math. 310, Amer. Math. Soc., Providence, RI, (2002), 1-24.

[3] K. Aker, S. Szabo. Algebraic Nahm transform for parabolic Higgs bundles on $\mathbb{P}^{1}$. Preprint math. AG/0610301.

[4] V. Balaji, I. Biswas, D. Nagaraj. Principal bundles over projective manifolds with parabolic structure over a divisor. Tohoku Math. J. 53 (2001), 337-367.

[5] P. Belkale. Local systems on $\mathbb{P}^{1}-S$ for $S$ a finite set. Compositio Math. 129 (2001), 67-86.

[6] B. Ben Hamed, L. Gavrilov. Families of Painlevé VI equations having a common solution. Int. Math. Res. Not. 60 (2005), 3727-3752.

[7] O. Biquard. Sur les fibrés paraboliques sur une surface complexe. J. London Math. Soc. 53 (1996), 302-316.

[8] I. Biswas. Parabolic bundles as orbifold bundles, Duke Math. J. 88 (1997), 305-325.

[9] I. Biswas. Flat connections on a punctured sphere and geodesic polygons in a Lie group. J. Geom. Phys. 39 (2001), 129-134.

[10] I. Biswas. Chern classes for parabolic bundles, J. Math. Kyoto Univ. 37 (1997), no. 4, 597-613.

[11] I. Biswas. Stable principal bundles and reduction of structure group. Preprint math. AG/0608569.

[12] I. Biswas, M. Mitra, S. Nag. Thurston boundary of Teichmller spaces and the commensurability modular group. Conform. Geom. Dyn. 3 (1999), 50-66.

[13] S. Bloch, H. Esnault. Local Fourier transforms and rigidity for $\mathcal{D}$-modules. Asian J. Math. 8 (2004), 587-605.

[14] P. Boalch. Painlevé equations and complex reflections. Proceedings of the Conference in Honor of F. Pham (Nice, 2002). Ann. Inst. Fourier 53 (2003), 1009-1022.

[15] P. Boalch. From Klein to Painlevé via Fourier, Laplace and Jimbo. Proc. London Math. Soc. 90 (2005), 167-208.

[16] H. Boden, K. Yokogawa. Moduli spaces of parabolic Higgs bundles and parabolic $K(D)$ pairs over smooth curves, I. Internat. J. Math. 7 (1996), 573-598. 
[17] A. Bolibruch. The Riemann-Hilbert problem on a compact Riemannian surface. Proc. Steklov Inst. Math. $\mathbf{2 3 8}$ (2002), 47-60.

[18] A. Bolibruch, S. Malek, C. Mitschi. On the generalized Riemann-Hilbert problem with irregular singularities. Expo. Math. 24 (2006), 235-272.

[19] N. Borne. Fibrés paraboliques et champ des racines. Int. Math. Res. Not. (2007) Vol. 2007 : article ID rnm049, 38 pages, doi:10.1093/imrn/rnm049.

[20] I. Bouw. Reduction of the Hurwitz space of metacyclic covers. Duke Math. J. 121 (2004), 75-111.

[21] S. Bradlow. Special metrics and stability for holomorphic bundles with global sections. J. Differential Geom. 33 (1991), 169-214.

[22] S. Bradlow, G. Daskalopolous. Moduli of stable pairs for holomorphic bundles over Riemann surfaces, II. Int. J. Math 4 (1993), 903-925.

[23] S. Bradlow, G. Daskalopolous, R. Wentworth. Birational equivalence of vortex moduli. Topology 35 (1996), 731-748.

[24] S. Bradlow, O. García-Prada, P. Gothen. Surface group representations and $U(p, q)$-Higgs bundles. J. Diff. Geom. 64 (2003), 111-170.

[25] N. Budur. Unitary local systems, multiplier ideals, and polynomial periodicity of Hodge numbers. Preprint math.AG/0610382.

[26] M. Burger, A. Iozzi, A. Wienhard. Surface group representations with maximal Toledo invariant. Preprint math.DG/0605656.

[27] C. Cadman. Using stacks to impose tangency conditions on curves. Amer. J. Math. 129 (2007), 405-427.

[28] M. Cailotto. Algebraic connections on logarithmic schemes, C. R. Acad. Sci. Paris Sér. I Math. 333 (2001), 1089-1094.

[29] D. Cohen, P. Orlik. Arrangements and local systems. Math. Res. Lett. 7 (2000), 299-316.

[30] K. Corlette. Flat G-bundles with canonical metrics. J. Diff. Geom. 28 (1988), 361-382.

[31] K. Corlette. Rigid representations of Kählerian fundamental groups. J. Diff. Geom. 33 (1991), 239-252.

[32] K. Corlette, C. Simpson. On the classification of rank two representations of quasiprojective fundamental groups. Compositio, to appear.

[33] W. Crawley-Boevey. On matrices in prescribed conjugacy classes with no common invariant subspace and sum zero. Duke Math. J. 118 (2003), 339-352.

[34] W. Crawley-Boevey. Indecomposable parabolic bundles and the existence of matrices in prescribed conjugacy class closures with product equal to the identity. Publ. Math. I.H.E.S. 100 (2004), 171-207.

[35] W. Crawley-Boevey, P. Shaw. Multiplicative preprojective algebras, middle convolution and the Deligne-Simpson problem. Adv. Math. 201 (2006), 180-208.

[36] M. Culler, P. Shalen. Varieties of group representations and splittings of 3-manifolds. Ann. of Math. 117 (1983), 109-146.

[37] G. Daskalopolous, S. Dostoglou, R. Wentworth. On the Morgan-Shalen compactification of the $S L(2, \mathbb{C})$ character varieties of surface groups. Duke Math. J. 101 (2000), 189-207. 
[38] G. Daskalopolous, S. Dostoglou, R. Wentworth. Character varieties and harmonic maps to R-trees. Math. Res. Lett. 5 (1998), 523-533.

[39] G. Daskalopoulos, R. Wentworth. (i) The Yang-Mills flow near the boundary of Teichmüller space. Math. Ann. 318 (2000), 1-42.

[40] P. Deligne. Equations différentielles a points singuliers reguliers. LECT. Notes IN MATH. 163, 1970.

[41] P. Deligne, G. Mostow. Commensurabilities among lattices in PU $(1, n)$. Ann. of Math. Studies 132, Princeton University Press (1993).

[42] M. Dettweiler. Galois realizations of classical groups and the middle convolution. Habilitationsschrift, Universität Heidelberg (2005). Preprint math. NT/0605381.

[43] M. Dettweiler, S. Reiter. On rigid tuples in linear groups of odd dimension. J. Algebra 222 (1999), 550-560.

[44] M. Dettweiler, S. Reiter. An algorithm of Katz and its application to the inverse Galois problem. Algorithmic methods in Galois theory. J. Symbolic Comput. 30 (2000), 761798.

[45] M. Dettweiler, S. Reiter. On the middle convolution. Preprint math. AG/0305311.

[46] M. Dettweiler, S. Reiter. Middle convolution of Fuchsian systems and the construction of rigid differential systems. J. Algebra 318 (2007), 1-24.

[47] M. Dettweiler, S. Reiter. Painlevé equations and the middle convolution. Adv. Geom. 7 (2007), 317-330.

[48] M. Dettweiler, S. Reiter. On exceptional rigid local systems. Preprint math. AG/0609142.

[49] S. Donaldson. A new proof of a theorem of Narasimhan and Seshadri. J. Diff. Geom. 18 (1983), 269-277.

[50] B. Dwork. On systems of ordinary differential equations with transcendental parameters. J. Differential Equations 156 (1999), 18-25.

[51] H. Esnault, C. Hertling. Semistable bundles on curves and reducible representations of the fundamental group. Int. J. Math. 12 (2001), 847-855.

[52] H. Esnault, E. Viehweg. Logarithmic De Rham complexes and vanishing theorems, Inventiones 86 (1986), 161-194.

[53] H. Esnault, E. Viehweg. Semistable bundles on curves and irreducible representations of the fundamental group. Algebraic geometry: Hirzebruch 70 (Warsaw, 1998) ConTEMP. MAтн. 241, AMS (1999), 129-138.

[54] A. Fujiki. Hyper-Kähler structure on the moduli space of flat bundles. Prospects in complex geometry (Katata and Kyoto, 1989) Springer LeCture Notes in Math. 1468 (1991), 1-83.

[55] O. Gleizer. Some explicit solutions of the additive Deligne-Simpson problem and their applications. Adv. Math. 178 (2003), 311-374.

[56] W. Goldman, E. Xia. Rank One Higgs bundles and representations of fundamental groups of Riemann surfaces. Mem. Amer. Math. Soc. 193 (2008), no. 904, viii+69 pp.

[57] V. Golyshev. Riemann-Roch variations. Izv. Math. 65 (2001), 853-881.

[58] A. Gorodentsev, S. Kuleshov. Helix theory. Mosc. Math. J. 4 (2004), 377-440, 535. 
[59] P. Gothen. Components of spaces of representations and stable triples. Topology 40 (2001), 823-850.

[60] M. Gromov, R. Schoen. Harmonic maps into singular spaces and $p$-adic superrigidity for lattices in groups of rank one. I.H.E.S. Publ. Math. 76 (1992), 165-246.

[61] Y. Haraoka. Evaluation of Stokes multipliers for a certain system of differential equations corresponding to a rigid local system. Funkcial. Ekvac. 46 (2003), 187-211.

[62] Y. Haraoka. Integral representations of solutions of differential equations free from accessory parameters. Adv. Math. 169 (2002), 187-240.

[63] Y. Haraoka, T. Yokoyama. Construction of rigid local systems and integral representations of their sections. Math. Nachr. 279 (2006), 255-271.

[64] Y. Haraoka, T. Yokoyama. On rigidity of Pfaffian systems coming from Okubo systems. Kyushu J. Math. 55 (2001), 189-205.

[65] T. Hausel. Compactification of moduli of Higgs bundles. J. Reine Angew. Math. 503 (1998), 169-192.

[66] T. Hausel, M. Thaddeus. Mirror symmetry, Langlands duality, and the Hitchin system. Inventiones 153 (2003), 197-229.

[67] M. Hien. Periods for irregular singular connections on surfaces. Periods for irregular singular connections on surfaces. Math. Ann. 337 (2007), 631-669.

[68] V. Hinich. Descent of Deligne groupoids. Internat. Math. Res. Notices 1997, 223-239.

[69] F. Hirzebruch. Arrangements of lines and algebraic surfaces. Arithmetic and geometry, Vol. II, Progr. MAth. 36 Birkhäuser (1983), 113-140.

[70] N. Hitchin. The self-duality equations on a Riemann surface. Proc. London Math. Soc. 55 (1987), 59-126.

[71] N. Hitchin. Poncelet polygons and the Painlevé equations. Geometry and analysis (Bombay, 1992), Tata Inst. Fund. Res., Bombay (1995), 151-185.

[72] N. Hitchin. Lie groups and Teichmüller space. Topology 31 (1992), 449-473.

[73] L. Illusie. Réduction semi-stable et décomposition de complexes de de Rham à coefficients, Duke Math. J. 60 (1990), 139-185.

[74] L. Illusie, K. Kato, C. Nakayama. Quasi-unipotent logarithmic Riemann-Hilbert correspondences, J. Math. Sci. Univ. Tokyo 12 (2005), 1-66.

[75] M. Inaba. Moduli of parabolic connections on a curve and Riemann-Hilbert correspondence. Preprint math. AG/0602004.

[76] M. Inaba, K. Iwasaki, Masa-Hiko Saito. Moduli of Stable Parabolic Connections, Riemann-Hilbert correspondence and Geometry of Painlevé equation of type VI, Part I. Publ. Res. Inst. Math. Sci. 42 (2006), 987-1089.

[77] J. Iyer, C. Simpson. A relation between the parabolic Chern characters of the de Rham bundles. Math. Ann. 338 (2007), 347-383.

[78] J. Jost, Y. Yang, K. Zuo. The cohomology of a variation of polarized Hodge structures over a quasi-compact Kähler manifold. J. Algebraic Geom. 16 (2007), 401-434.

[79] J. Jost, Y. Yang, K. Zuo. Cohomologies of unipotent harmonic bundles over noncompact curves. J. Reine Angew. Math. 609 (2007), 137-159. 
[80] M. Kapranov. Injective resolutions of $B G$ and derived moduli spaces of local systems. $J$. Pure Appl. Algebra 155 (2001), 167-179.

[81] F. Kato. The relative log Poincaré lemma and relative log de Rham theory. Duke Math. J., 93 (1998), 179-206.

[82] K. Kato, C. Nakayama. Log Betti cohomology, log étale cohomology, and log de Rham cohomology of log schemes over $\mathbb{C}$. Kodai Math. J. 22 (1999), 161-186.

[83] N. Katz, T. Oda. On the differentiation of de Rham cohomology classes with respect to parameters. J. Math. Kyoto Univ. 8, 2 (1968), 199-213.

[84] N. Katz. The regularity theorem in algebraic geometry. Actes du Congrés International des Mathématiciens (Nice, 1970), Tome 1, Gauthier-Villars, Paris, (1971), 437-443.

[85] N. Katz. Exponential Sums and Differential Equations. Annals of Mathematics StudIES 124, Princeton University Press (1990).

[86] N. Katz. Rigid local systems. Annals of Mathematics Studies 139, Princeton University Press (1996).

[87] H. Konno. Construction of the moduli space of stable parabolic Higgs bundles on a Riemann surface. J. Math. Soc. Japan 45 (1993), 253-276.

[88] N. Korevaar, R. Schoen. Sobolev spaces and harmonic maps for metric space targets. Comm. Anal. Geom. 1 (1993), 561-659.

[89] V. Kostov. On the Deligne-Simpson problem. C.R.A.S. 329 (1999), 657-662.

[90] V. Kostov. The Deligne-Simpson problem for zero index of rigidity. Perspectives of complex analysis, differential geometry and mathematical physics (St. Konstantin, 2000), World Sci. Publ., (2001), 1-35.

[91] V. Kostov. On the Deligne-Simpson problem. Proc. Steklov Inst. Math. 238 (2002), 148185.

[92] V. Kostov. On some aspects of the Deligne-Simpson problem. J. Dynam. Control Systems 9 (2003), 393-436.

[93] V. Kostov. On the Deligne-Simpson problem and its weak version. Bull. Sci. Math. 128 (2004), 105-125.

[94] V. Kostov. The Deligne-Simpson problem-a survey. J. Algebra 281 (2004), 83-108.

[95] V. Kostov. The connectedness of some varieties and the Deligne-Simpson problem. J. Dyn. Control Syst. 11 (2005), 125-155.

[96] G. Laumon. Exponential sums and l-adic cohomology: a survey. Israel J. Math. 120 (2000), 225-257.

[97] G. Laumon. La transformation de Fourier géométrique et ses applications. Proceedings of ICM, Kyoto 1990, Math. Soc. Japan (1991), 437-445.

[98] R. Lawrence. Homological representations of the Hecke algebra. Comm. Math. Phys. 135 (1990), 141-191.

[99] Jiayu Li. Hermitian-Einstein metrics and Chern number inequalities on parabolic stable bundles over Kähler manifolds. Comm. Anal. Geom. 8 (2000), 445-475.

[100] J. Li, M.S. Narasimhan. Hermitian-Einstein metrics on parabolic stable bundles. Acta Math. Sin. 15 (1999), 93-114. 
[101] Jiayu Li, M. S. Narasimhan. A note on Hermitian-Einstein metrics on parabolic stable bundles. Acta Math. Sinica 17 (2001), 77-80.

[102] R. Litcanu. Lamé operators with finite monodromy - a combinatorial approach. J. Differential Equations 207 (2004), 93-116.

[103] A. Lubotzky, A. Magid. Varieties of representations of finitely generated groups. MEM. Amer. Math. Soc. 58 (1985).

[104] Y. Manin. Moduli Fuchsiani, Annali Scuola Normale Sup. di Pisa Ser. III 19 (1965), 113-126.

[105] I. Marin. Caractérisations de la représentation de Burau. Expo. Math. 21 (2003), 263-278.

[106] E. Markman, E. Xia. The moduli of flat PU $(p, p)$-structures with large Toledo invariants. Math. Z. 240 (2002), 95-109.

[107] M. Maruyama, K. Yokogawa. Moduli of parabolic stable sheaves. Math. Ann. 293 (1992), no. $1,77-99$.

[108] K. Matsuki, M. Olsson. Kawamata-Viehweg vanishing and Kodaira vanishing for stacks. Math. Res. Lett. 12 (2005), 207-217.

[109] M. Mehta. Birational equivalence of Higgs moduli. Internat. J. Math. 16 (2005), 365-386.

[110] T. Mochizuki. Asymptotic behaviour of tame nilpotent harmonic bundles with trivial parabolic structure. J. Diff. Geom. 62 (2002), 351-559.

[111] T. Mochizuki. Asymptotic behaviour of tame harmonic bundles and an application to pure twistor D-modules. I, II. Mem. Amer. MATh. Soc. 185 (2007), nos. 869-870, xii+565 pp.

[112] Mochizuki, T. Kobayashi-Hitchin correspondence for tame harmonic bundles and an application. Astérisque 309 (2006), viii+117 pp.

[113] B. Moishezon, M. Teicher. Braid group technique in complex geometry. I. Line arrangements in $\mathbb{C P}^{2}$. Braids (Santa Cruz, 1986), Contemp. Math. 78, A.M.S. (1988), 425-555.

[114] H. Nakajima. Hyper-Kähler structures on moduli spaces of parabolic Higgs bundles on Riemann surfaces. Moduli of vector bundles (Sanda, Kyoto, 1994) LECT. Notes Pure Appl. Матн. 179 (1996), 199-208.

[115] O. Neto, P. Silva. On regular holonomic systems with solutions ramified along $y^{k}=x^{n}$. Pacific J. Math. 207 (2002), 463-487.

[116] N. Nitsure. Moduli space of semistable pairs on a curve. Proc. London Math. Soc. 62 (1991), 275-300.

[117] N. Nitsure. Moduli of semistable logarithmic connections. J. Amer. Math. Soc. 6 (1993), 597-609.

[118] B. Noohi. Fundamental groups of algebraic stacks. J. Inst. Math. Jussieu 3 (2004), 69-103.

[119] Ohtsuki, M. A residue formula for Chern classes associated with logarithmic connections, Tokyo J. Math. 5 (1982), no. 1, 13-21.

[120] D. Panov. Polyhedral Kähler manifolds. Doctoral thesis, Ecole Polytechnique (2005).

[121] A. Reznikov. Continuous cohomology of the group of volume-preserving and symplectic diffeomorphisms, measurable transfer and higher asymptotic cycles. Selecta Math. $\mathbf{5}$ (1999), 181-198. 
[122] A. Reznikov. The structure of Kähler groups. I. Second cohomology. Motives, polylogarithms and Hodge theory, Irvine, 1998, Int. Press LeCt. Ser. 3 (II) (2002), 717-730.

[123] D. Roberts. Rigid Jordan tuples. Preprint available at http://cda.morris.umn.edu/ ${ }^{\sim}$ roberts/.

[124] C. Sabbah. Harmonic metrics and connections with irregular singularities. Ann. Inst. Fourier 49 (1999), 1265-1291.

[125] C. Sabbah. Polarizable twistor D-modules. AstéRISque 300, (2005).

[126] A. Schmitt. Projective moduli for Hitchin pairs. Internat. J. Math. 9 (1998), 107-118.

[127] C. S. Seshadri. Moduli of vector bundles on curves with parabolic structures. Bull. Amer. Math. Soc. 83 (1977), 124-126.

[128] C. Simpson. Constructing variations of Hodge structure using Yang-Mills theory and applications to uniformization. J. Amer. Math. Soc. 1 (1988), 867-918.

[129] C. Simpson. Harmonic bundles on noncompact curves. J. Amer. Math. Soc. 3 (1990), 713-770.

[130] C. Simpson. Products of matrices. Differential geometry, global analysis, and topology (Halifax, 1990), CMS Conf. Proc. 12 (1991), 157-185.

[131] B. Steer, A. Wren. The Donaldson-Hitchin-Kobayashi correspondence for parabolic bundles over orbifold surfaces. Canad. J. Math. 53 (2001), 1309-1339.

[132] K. Strambach, H. Völklein. On linearly rigid tuples. J. Reine Angew. Math. 510 (1999), 57-62.

[133] S. Szabo. Nahm transform for integrable connections on the Riemann sphere. Preprint math.DG/0511471. To appear, Mémoires de la S.M.F.

[134] M. Thaddeus. Variation of moduli of parabolic Higgs bundles. J. Reine Angew. Math. 547 (2002), 1-14.

[135] I. Tuba, H. Wenzl. Representations of the braid group $B_{3}$ and of $\operatorname{SL}(2, Z)$. Pacific J. Math. 197 (2001), 491-510.

[136] M. van der Put. Recent work on differential Galois theory. Séminaire Bourbaki 1997/98. Astérisque 252 (1998), Exp. No. 849, 341-367.

[137] M. van der Put. Grothendieck's conjecture for the Risch equation $y^{\prime}=a y+b$. Indag. Math. (N.S.) 12 (2001), 113-124.

[138] H. Völklein. A transformation principle for covers of $\mathbb{P}^{1}$. J. Reine Angew. Math. 534 (2001), 156-168.

[139] H. Völklein. The braid group and linear rigidity. Geom. Dedicata 84 (2001), 135-150.

[140] E. Xia. The moduli of flat $U(p, 1)$ structures on Riemann surfaces. Geom. Dedicata 97 (2003), 33-43.

[141] K. Yokogawa. Compactification of moduli of parabolic sheaves and moduli of parabolic Higgs sheaves. J. Math. Kyoto Univ. 33 (1993), 451-504.

[142] K. Yokogawa. Infinitesimal deformation of parabolic Higgs sheaves. Internat. J. Math. 6 (1995), 125-148.

[143] T. Yokoyama. Construction of systems of differential equations of Okubo normal form with rigid monodromy. Math. Nachr. 279 (2006), 327-348. 
Carlos Simpson

CNRS, Laboratoire J. A. Dieudonné, UMR 6621

Université de Nice-Sophia Antipolis

Parc Valrose

06108 Nice, Cedex 2, France

E-mail: carlos@math.unice.fr

$U R L:$ http://math.unice.fr/ carlos/ 